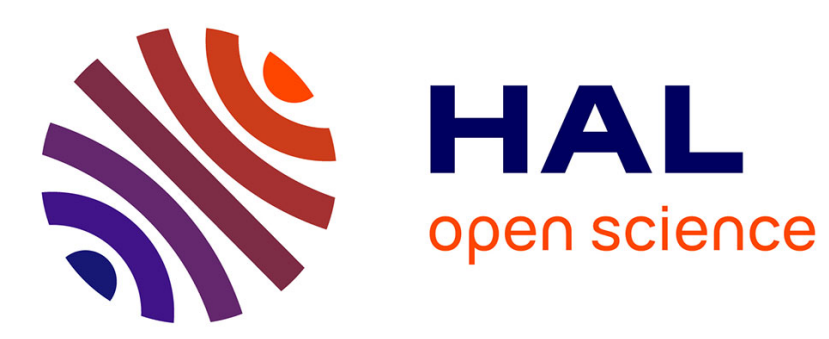

\title{
A Godunov-type method for the seven-equation model of compressible two-phase flow
}

\author{
Annalisa Ambroso, Christophe Chalons, Pierre-Arnaud Raviart
}

\section{To cite this version:}

Annalisa Ambroso, Christophe Chalons, Pierre-Arnaud Raviart. A Godunov-type method for the seven-equation model of compressible two-phase flow. 2010. hal-00517375

\section{HAL Id: hal-00517375 \\ https://hal.science/hal-00517375}

Preprint submitted on 14 Sep 2010

HAL is a multi-disciplinary open access archive for the deposit and dissemination of scientific research documents, whether they are published or not. The documents may come from teaching and research institutions in France or abroad, or from public or private research centers.
L'archive ouverte pluridisciplinaire HAL, est destinée au dépôt et à la diffusion de documents scientifiques de niveau recherche, publiés ou non, émanant des établissements d'enseignement et de recherche français ou étrangers, des laboratoires publics ou privés. 


\title{
A Godunov-type method for the seven-equation model of compressible two-phase flow
}

\author{
A. Ambroso*, C. Chalons ${ }^{\dagger}$ P.-A. Raviart ${ }^{\ddagger}$
}

April 26, 2010

\begin{abstract}
We are interested in the numerical approximation of the solutions of the compressible seven-equation two-phase flow model. We propose a numerical srategy based on the derivation of a simple, accurate and explicit approximate Riemann solver. The source terms associated with the external forces and the drag force are included in the definition of the Riemann problem, and thus receive an upwind treatment. The objective is to try to preserve, at the numerical level, the asymptotic property of the solutions of the model to behave like the solutions of a drift-flux model with an algebraic closure law when the source terms are stiff. Numerical simulations and comparisons with other strategies are proposed.
\end{abstract}

\section{Contents}

1 Introduction

2 The governing equations and some mathematical properties 4

2.1 Model . . . . . . . . . . . . . . . . . . . 4

2.2 Closure relations . . . . . . . . . . . . . . . . 6

2.3 Asymptotic properties ................ 8

3 The principle of the numerical method 9

4 The approximate Riemann solver 12

4.1 Generalities . . . . . . . . . . . . . . . . 12

4.1.1 Consistency with the integral form of $(25) \ldots . . . . .13$

4.1.2 The Godunov-type method . . . . . . . . . . . . . . . 14

4.2 Construction of the Riemann solver . . . . . . . . . . . . . . . . 15

4.2.1 First notations and form of the approximate Riemann solver 16

4.2.2 The consistency relations . . . . . . . . . . . . . 17

4.2.3 Definition of the intermediate states . . . . . . . . . . 18

*AREVA-DRI, Paris, France. Email : annalisa.ambroso@areva.com

${ }^{\dagger}$ CEA-Saclay, DEN/DANS/DM2S/SFME/LETR, F-91191 Gif-sur-Yvette, France. Email : chalons@math.jussieu.fr

${ }^{\ddagger}$ UPMC Univ Paris 06, UMR 7598, Laboratoire Jacques-Louis Lions, Paris, F-75005, France; CNRS, UMR 7598 LJLL, Paris, F-75005, France. Email : pa@raviart.com 
5 The numerical scheme $\quad 23$

5.1 The first step: $\mathbf{U}^{n} \rightarrow \mathbf{U}^{n+1 / 2} \ldots \ldots \ldots \ldots 24$

5.2 The second step: $\mathbf{U}^{n+1 / 2} \rightarrow \mathbf{U}^{n+1} \ldots \ldots \ldots 26$

6 Properties of the scheme and open problems $\quad 27$

7 Numerical results $\quad 28$

7.1 Riemann problems . . . . . . . . . . . . . . . . . . . 29

7.1.1 Test 1 : isolated coupling wave . . . . . . . . . . . 29

7.1.2 Test 2: a general Riemann problem . . . . . . . . . 32

7.1.3 Test 3 : a general Riemann problem with several values of $\chi \ldots \ldots \ldots \ldots \ldots \ldots \ldots$

7.2 Influence of the source terms . . . . . . . . . . . . . . . 39

7.2.1 Test 4 : the Ransom water faucet problem . . . . . . . . 39

7.2.2 Test 5 : the sedimentation test case . . . . . . . . . . 41

7.2.3 Test 6 : velocities desequilibrium . . . . . . . . . . . . . . 44

7.2.4 Test 7 : vertical bubbly column . . . . . . . . . . . . 44

\section{Introduction}

We are interested in the computation of compressible two-phase flows. There are essentially two classes of models for describing such flows. The first class consists in considering the two-phase fluid as a mixture and treating it as a single fluid with a fairly complex thermodynamics. The second class, the two-fluid models, treats each phase as a separate fluid and consists in writing balance equations for the mass, momentum and energy of each phase, together with exchanging terms between the two phases. We will consider here a particular two-fluid model, namely the so-called two fluid-two pressure or seven-equation model. It was first proposed in Baer \& Nunziato [6] for granular energetic combustible materials embedded in gaseous combustion products. Its mathematical properties were first comprehensively studied in Embid \& Baer [16]. Then, the model (and related ones, see among others Stewart \& Wendroff [41], Abgrall \& Saurel [37], [38]...) has gained interest for the modelling and computation of two phase flows. See for instance, in a non-exhaustive way, Kapila et al. [29], Glimm et al. [23], Abgrall \& Saurel [37], Gavrilyuk \& Saurel [22], Gallouët, Hérard \& Seguin [21], Coquel, Gallouët, Hérard \& Seguin [13], and more recently Ambroso, Chalons, Coquel \& Galié [1], Tokareva \& Toro [42], and the references therein.

One of the main features of this model is to involve two distinct velocities $u_{1}$ and $u_{2}$ and two pressures $p_{1}$ and $p_{2}$ associated with the two phases. It is actually more common, at least in the meantime and in the nuclear industry, to use two fluid-one pressure models where both phases share the same pressure $p=p_{1}=p_{2}$. This approach is justified by the very short time-scale linked with the phenomenon of relaxation of the two pressures towards an equilibrium. However, the partial differential equations system corresponding to this assumption lacks some good mathematical properties : eigenvalues of the Jacobian matrix are not always real but may take complex values. The Cauchy problem for this 
system is then generally ill-posed. On the contrary, the two fluid-two pressure seven-equation model admits systematically seven real eigenvalues and is seen to have a basis of right eigenvectors, at least in the context of subsonic flows that will be considered throughout the present paper. The hyperbolicity property thus makes the two-fluid two-pressure approach very attractive in comparison to models using a pressure equilibrium assumption.

From a numerical point of view, the seven-equation model is not easy to deal with for several reasons. The first difficulty is related to the large size of the model : it is made of seven equations in one space dimension. As an immediate consequence, the Riemann problem associated with the convective part involves a large number of intermediate states (six generally speaking) and is difficult to determine or even approximate, and so is the derivation of Godunovtype method. The second difficulty comes from the presence of nonconservative products in the governing equations and more precisely the fact that generally speaking, the model does not admit any equivalent conservation form (actually, the nonconservative terms vanish in the very particular situation where the void fractions $\alpha_{k}$ are locally constant in space and the structure of the model becomes the one of two (decoupled) classical gas dynamics systems). Note however that here, the nonconservative products are associated with a linearly degenerate characteristic field so that by contrast with nonconservative products appearing in shocks (see for instance LeFloch [32], [33]), discrete solutions are not expected to depend on the underlying numerical viscosity (see Guillemaud [25]). Difficulties may arise but they are linked with the appearance of resonance phenomena (see Andrianov \& Warnecke [5], Andrianov [3]...). At last but not least, the closure relations associated with the pressure laws $p_{k}, k=1,2$ may be strongly nonlinear in practice which renders even more difficult the derivation of exact or approximate Riemann solvers.

A lot of papers are devoted to the numerical resolution of two-fluid two-pressure models and the question of how to discretize the nonconservative terms. In particular, the following is certainly not exhaustive. Let us first mention that Saurel \& Abgrall [37] and Andrianov, Saurel \& Warnacke [4] for instance (see also Saurel \& Lemetayer [39] for a multidimensional framework) take into account the nonconservative terms by means of a free streaming physical condition associated with uniform velocity and pressure profiles. The discretization technique of [37] is improved by the same authors in [38]. Then, in Andrianov \& Warnecke [5] and Schwendeman, Wahle \& Kapila [40], the common objective is to get exact solutions for the Riemann problem of the model. The approach is inverse in [5] in the sense that the initial left and right states are obtained as function of the intermediate states of the solution. On the contrary, a direct iterative approach is used in [40] leading to exact solutions of the Riemann problem for any initial left and right states. See also the recent work of Deledicque \& Papalexandris [15]. Another direct approach to construct theoretical solutions is proposed in Castro \& Toro [9]. In this work the authors propose to solve the Riemann problem approximately assuming that all the nonlinear characteristic fields are associated with rarefaction waves. More recently, Tokareva \& Toro propose in [42] a HLLC-type approximate Riemann solver which takes into account all the seven waves that are naturally present in the model, and that can be seen as a similar but faster approach in comparison to the exact solver proposed in [40]. Finally, all these (approximate or exact) solutions are used to develop a Godunov-type method. At last, other finite volumes methods have 
been used. For instance in Gallouët et al. [21] (see also Guillemaud [25]), the approximation of the convective terms of the system is based on the Rusanov scheme (Rusanov [36]) and the so-called VFRoe-ncv scheme (Buffard et al. [8]), these strategies being adapted to the nonconservative framework. In Munkejord [34] and Karni et al. [30], the author use Roe-type schemes.

Many of the above mentioned methods are only devoted to the convective part of the seven-equation model, focusing in addition on a specific choice of the interfacial velocity $u_{I}$ naturally present in the governing equations. They are often based on an exact or approximate resolution of the complete Riemann problem, leading to fully nonlinear algorithms. In this context, our objective here is to derive the simplest possible approximate Riemann solver for the seven-equation model, which is accurate, explicit and well-adapted for the nuclear industry. By construction, it will be able to deal with any admissible interfacial velocity $u_{I}$, and any (possibly strongly nonlinear) closure relations for the pressure laws $p_{k}$, $k=1,2$. It will also enjoy several pleasant stability properties. Importantly, the definition of our approximate Riemann solver will also include the usual source terms associated with the gravity field and the drag force. The objective is to propose an upwind treatment of the sources, so as to preserve the asymptotic behavior of the solutions in the asymptotic regime of relaxation time-scales tending to zero (see section 2.3 for details). Proving the so-called asymptoticpreserving property of the proposed method unfortunately still remains an open problem. In that sense, this study can be understood as a first attempt to design asymptotic-preserving schemes for the seven-equation model.

The outline of the paper is as follows. Section 2 introduces some notations, gives the governing equations of the two-fluid two-pressure model and states its basic properties. Section 3 proposes a rough description of the whole numerical scheme. Next, section 4 describes the approximate Riemann solver and section 5 details the numerical scheme. At last, section 6 gives the stability properties satisfied by the scheme and states some open problems. Section 7 is devoted to numerical experiments.

\section{The governing equations and some mathemat- ical properties}

\section{$2.1 \quad$ Model}

In one space dimension, the model under consideration reads as follows :

$$
\left\{\begin{array}{l}
\frac{\partial \alpha_{k}}{\partial t}+u_{I} \frac{\partial \alpha_{k}}{\partial x}=\Theta\left(p_{k}-p_{l}\right) \\
\frac{\partial}{\partial t}\left(\alpha_{k} \varrho_{k}\right)+\frac{\partial}{\partial x}\left(\alpha_{k} \varrho_{k} u_{k}\right)=0 \\
\frac{\partial}{\partial t}\left(\alpha_{k} \varrho_{k} u_{k}\right)+\frac{\partial}{\partial x}\left(\alpha_{k}\left(\varrho_{k} u_{k}^{2}+p_{k}\right)\right)-p_{I} \frac{\partial \alpha_{k}}{\partial x}=\alpha_{k} \varrho_{k} g-K\left(u_{k}-u_{l}\right), \\
\frac{\partial}{\partial t}\left(\alpha_{k} \varrho_{k} e_{k}\right)+\frac{\partial}{\partial x}\left(\alpha_{k}\left(\varrho_{k} e_{k}+p_{k}\right) u_{k}\right)-p_{I} u_{I} \frac{\partial \alpha_{k}}{\partial x}= \\
=\alpha_{k} \varrho_{k} g u_{k}-p_{I} \Theta\left(p_{k}-p_{l}\right)-u_{I} K\left(u_{k}-u_{l}\right)
\end{array}\right.
$$


where $k, l=1,2, l \neq k$ with the constraint

$$
\alpha_{1}+\alpha_{2}=1,
$$

meaning that the fluids are unmixable. In the above equations, $\alpha_{k}, \varrho_{k}, u_{k}, e_{k}$ and $p_{k}$ denote the volume fraction, density, velocity, specific total energy and pressure of the phase $k, k=1,2$, while $g$ stands for the gravity acceleration. The structure of the convective part of system (1) is the one of two gas dynamics systems for each phase, coupled with a transport equation with velocity $u_{I}$ for the void fraction $\alpha_{k}$. We note however that non-conservative products involving the interfacial pressure $p_{I}$ and velocity $u_{I}$ (to be precised later on) and the space derivative of the void fractions $\alpha_{k}$ are naturally present in the equations. These terms act as coupling terms in the evolution of the two phases in the sense that if they were not present (in case $\frac{\partial \alpha_{k}}{\partial x}=0$ for instance), the phase evolutions would decouple and the usual conservative form of the gas dynamics equations would be recovered. In the general setting, we thus notice that system (1) is of the general nonconservative form

$$
\frac{\partial \mathbf{U}}{\partial t}+\frac{\partial}{\partial x} \mathbf{F}(\mathbf{U})+\mathbf{B}(\mathbf{U}) \frac{\partial \mathbf{U}}{\partial x}=\mathbf{S}(\mathbf{U})
$$

where

$$
\begin{gathered}
\mathbf{U}=\left(\begin{array}{c}
\alpha_{1} \\
\mathbf{U}_{1} \\
\mathbf{U}_{2}
\end{array}\right), \quad \mathbf{U}_{k}=\left(\begin{array}{c}
\alpha_{k} \varrho_{k} \\
\alpha_{k} \varrho_{k} u_{k} \\
\alpha_{k} \varrho_{k} e_{k}
\end{array}\right), \\
\mathbf{F}(\mathbf{U})=\left(\begin{array}{c}
0 \\
\mathbf{F}_{1}\left(\mathbf{U}_{1}\right) \\
\mathbf{F}_{2}\left(\mathbf{U}_{2}\right)
\end{array}\right), \quad \mathbf{F}_{k}\left(\mathbf{U}_{k}\right)=\left(\begin{array}{c}
\alpha_{k} \varrho_{k} u_{k} \\
\alpha_{k}\left(\varrho_{k} u_{k}^{2}+p_{k}\right) \\
\alpha_{k}\left(\varrho_{k} e_{k}+p_{k}\right) u_{k}
\end{array}\right), \\
\mathbf{S}_{k}(\mathbf{U})=\left(\begin{array}{c}
\Theta\left(p_{1}-p_{2}\right) \\
\mathbf{S}_{1}(\mathbf{U}) \\
\mathbf{S}_{2}(\mathbf{U}) \\
0 \\
\mathbf{S}(\mathbf{U})= \\
\alpha_{k} \varrho_{k} g-K\left(u_{k}-u_{l}\right) \\
\alpha_{k} \varrho_{k} g u_{k}-p_{I} \Theta\left(p_{k}-p_{l}\right)-u_{I} K\left(u_{k}-u_{l}\right)
\end{array}\right),
\end{gathered}
$$


$k, l=1,2, l \neq k$, and the $7 \times 7$ matrix $\mathbf{B}(\mathbf{U})$ is given by

$$
\mathbf{B}(\mathbf{U})=\left(\begin{array}{cl}
u_{I} & \vdots \\
0 & \vdots \\
-p_{I} & \vdots \\
-p_{I} u_{I} & \vdots \\
0 & \vdots \\
p_{I} & \vdots \\
p_{I} u_{I} & \vdots
\end{array}\right) .
$$

\subsection{Closure relations}

In order to close the system, we supplement (1),(2) with closure relations that we now describe in details. It amounts to define the pressure laws $p_{k}$, the pressure relaxation coefficient $\Theta$, the drag force coefficient $K$, and the interfacial velocity $u_{I}$ and pressure $p_{I}$. We note in particular that we have decided to neglect the dissipation terms due to laminar or turbulent viscosity and heat conduction. Moreover, for the sake of simplicity, phase changes and heat exchanges between the two phases are not taken into account.

On the one hand, each phase is provided with an equation of state

$$
p_{k}=p_{k}\left(\varrho_{k}, \varepsilon_{k}\right), \quad k=1,2,
$$

where $\varepsilon_{k}=e_{k}-\frac{u_{k}^{2}}{2}$ is the specific internal energy. On the other hand $\Theta$ will be typically chosen of the form

$$
\Theta=\frac{1}{\tau_{p}} \frac{\alpha_{1} \alpha_{2}}{p_{1}+p_{2}}
$$

where $\tau_{p}$ is the pressure relaxation time-scale, while $K$ will be set to be of the classical form

$$
K=\frac{1}{8} C_{D} a^{i n t} \rho_{1}\left|u_{1}-u_{2}\right|,
$$

in the numerical simulations (see section 7 for the definition of the related coefficients). Other choices are of course possible, we refer for instance the reader to Ishii \& Zuber [28].

It remains to specify $u_{I}$ and $p_{I}$. With this in mind, we follow [21] and first observe that the eigenvalues of the Jacobian matrix $\mathbf{F}^{\prime}(\mathbf{U})+\mathbf{B}(\mathbf{U})$ of (1) are always real and given by $u_{I}, u_{k}, u_{k} \pm c_{k}, k=1,2$, where $c_{k}$ denotes the sound speed of the phase $k$. The system (1) turns out to be only weakly hyperbolic, since the hyperbolicity property itself fails when $u_{I}=u_{k} \pm c_{k}$ for some index $k$, i.e. resonance occurs. When the system (1) is hyperbolic, one can easily check that for $k=1,2$, and similarly to the classical gas dynamics equations, the characteristic fields associated with the eigenvalues $u_{k} \pm c_{k}$ are genuinely nonlinear while the one associated with $u_{k}$ is linearly degenerate. As far as the characteristic field associated with $u_{I}$ is concerned, it is usually required to be linearly degenerate in practice. This property holds as soon as

$$
u_{I}=\beta u_{1}+(1-\beta) u_{2}, \quad \beta=\frac{\chi \alpha_{1} \varrho_{1}}{\chi \alpha_{1} \varrho_{1}+(1-\chi) \alpha_{2} \varrho_{2}}
$$


where $\chi \in[0,1]$ is a constant, which allows to define the interfacial velocity $u_{I}$. The usual choices for $\chi$ are $0, \frac{1}{2}, 1$. Regarding the interfacial pressure $p_{I}$, we set

$$
p_{I}=\mu p_{1}+(1-\mu) p_{2}, \quad \mu=\mu(\mathbf{U}) \in[0,1] .
$$

The choice of the coefficient $\mu$ is based on entropy considerations. If we denote by $s_{k}=s_{k}\left(\varrho_{k}, \varepsilon_{k}\right)$ the specific entropy of the phase $k$, it is an easy matter to check that any sufficiently smooth solution of (1) satisfies

$$
\left\{\begin{array}{l}
\frac{\partial}{\partial t}\left(\sum_{k=1}^{2} \alpha_{k} \varrho_{k} s_{k}\right)+\frac{\partial}{\partial x}\left(\sum_{k=1}^{2} \alpha_{k} \varrho_{k} s_{k} u_{k}\right)+\sum_{k=1}^{2} \frac{1}{T_{k}}\left(p_{I}-p_{k}\right)\left(u_{k}-u_{I}\right) \frac{\partial \alpha_{k}}{\partial x}= \\
=\sum_{k=1}^{2} \frac{1}{T_{k}}\left\{K\left(u_{l}-u_{k}\right)\left(u_{I}-u_{k}\right)+\Theta\left(p_{l}-p_{k}\right)\left(p_{I}-p_{k}\right)\right\}
\end{array}\right.
$$

where $T_{k}$ is the temperature of the phase $k$. Since by (12) and (13)

$$
\left\{\begin{array}{l}
\sum_{k=1}^{2} \frac{1}{T_{k}}\left(K\left(u_{l}-u_{k}\right)\left(u_{I}-u_{k}\right)+\Theta\left(p_{l}-p_{k}\right)\left(p_{I}-p_{k}\right)\right)= \\
=\left(\frac{1-\beta}{T_{1}}+\frac{\beta}{T_{2}}\right) K\left(u_{2}-u_{1}\right)^{2}+\left(\frac{1-\mu}{T_{1}}+\frac{\mu}{T_{2}}\right) \Theta\left(p_{2}-p_{1}\right)^{2} \geq 0,
\end{array}\right.
$$

we obtain

$$
\left\{\begin{array}{l}
\frac{\partial}{\partial t}\left(\sum_{k=1}^{2} \alpha_{k} \varrho_{k} s_{k}\right)+\frac{\partial}{\partial x}\left(\sum_{k=1}^{2} \alpha_{k} \varrho_{k} s_{k} u_{k}\right)= \\
=\left(\frac{1-\beta}{T_{1}}+\frac{\beta}{T_{2}}\right) K\left(u_{2}-u_{1}\right)^{2}+\left(\frac{1-\mu}{T_{1}}+\frac{\mu}{T_{2}}\right) \Theta\left(p_{2}-p_{1}\right)^{2} \geq 0
\end{array}\right.
$$

provided that we have

$$
\frac{1}{T_{1}}\left(p_{I}-p_{1}\right)\left(u_{1}-u_{I}\right)-\frac{1}{T_{2}}\left(p_{I}-p_{2}\right)\left(u_{2}-u_{I}\right)=0
$$

which amounts to choose

$$
\mu=\mu(\beta)=\frac{(1-\beta) T_{2}}{\beta T_{1}+(1-\beta) T_{2}} .
$$

Then, given a monotonically decreasing $C^{1}$ function $\phi=\phi(s)$, we set

$$
\eta=\eta(\mathbf{U})=\sum_{k=1}^{2} \alpha_{k} \varrho_{k} \phi\left(s_{k}\right), \quad q=q(\mathbf{U})=\sum_{k=1}^{2} \alpha_{k} \varrho_{k} \phi\left(s_{k}\right) u_{k} .
$$

Under the condition (12), we have for any sufficiently smooth solution of (1)

$$
\left\{\begin{array}{l}
\frac{\partial \eta}{\partial t}+\frac{\partial q}{\partial x}=\left(\frac{(1-\beta) \phi^{\prime}\left(s_{1}\right)}{T_{1}}+\frac{\beta \phi^{\prime}\left(s_{2}\right)}{T_{2}}\right) K\left(u_{2}-u_{1}\right)^{2}+ \\
+\left(\frac{(1-\mu) \phi^{\prime}\left(s_{1}\right)}{T_{1}}+\frac{\mu \phi^{\prime}\left(s_{2}\right)}{T_{2}}\right) \Theta\left(p_{2}-p_{1}\right)^{2} \leq 0
\end{array}\right.
$$


so that $(\eta, q)$ is a mathematical entropy pair if in addition the function $\phi$ is convex. A weak solution of (1) (in a sense to be precised) should then satisfy the entropy inequality

$$
\left\{\begin{array}{l}
\frac{\partial \eta}{\partial t}+\frac{\partial q}{\partial x} \leq\left(\frac{(1-\beta) \phi^{\prime}\left(s_{1}\right)}{T_{1}}+\frac{\beta \phi^{\prime}\left(s_{2}\right)}{T_{2}}\right) K\left(u_{2}-u_{1}\right)^{2}+ \\
+\left(\frac{(1-\mu) \phi^{\prime}\left(s_{1}\right)}{T_{1}}+\frac{\mu \phi^{\prime}\left(s_{2}\right)}{T_{2}}\right) \Theta\left(p_{2}-p_{1}\right)^{2} \leq 0
\end{array}\right.
$$

The system is now closed thanks to the relations (9), (10), (11), (12), (13) and (15).

\subsection{Asymptotic properties}

The objective of this section is to mention some asymptotic properties satisfied by the solutions of (1). For that, we assume that the time-scales involved in the pressure relaxation and drag force coefficients are much smaller than the other characteristic time-scales of the system. More precisely, we assume that

$$
\Theta(\mathbf{U})=\frac{\theta(\mathbf{U})}{\varepsilon^{2}}, \quad K(\mathbf{U})=\Lambda(\mathbf{U})\left|u_{2}-u_{1}\right|, \quad \Lambda(\mathbf{U})=\frac{\lambda(\mathbf{U})}{\varepsilon^{2}}
$$

for a small parameter $\varepsilon>0$ which implies that

$$
p_{2}-p_{1}=O\left(\varepsilon^{2}\right), \quad u_{2}-u_{1}=O(\varepsilon) .
$$

In other words, this means that the pressures relax to the equilibrium twice faster than the velocities. Then, one can prove as $\varepsilon$ tends to 0 that (1) admits an asymptotic limit, the so-called differential drift-flux limit that we now briefly recall for the sake of completeness. We refer for instance the reader to [2], [14], [24], and the references therein for more details. Let us define the following mixture quantities : density $\varrho$, velocity $u$, pressure $p$ and total energy $e$ by

$$
\varrho=\sum_{k=1}^{2} \alpha_{k} \varrho_{k}, \quad \varrho u=\sum_{k=1}^{2} \alpha_{k} \varrho_{k} u_{k}, \quad p=\sum_{k=1}^{2} \alpha_{k} p_{k}, \quad \varrho e=\sum_{k=1}^{2} \alpha_{k} \varrho_{k} e_{k} .
$$

We introduce in addition the relative velocity $u_{r}$, and the mass fraction $Y$ of the phase 2 :

$$
u_{r}=u_{2}-u_{1}, \quad Y=\frac{\alpha_{2} \varrho_{2}}{\varrho} .
$$

Then, following the classical Chapman-Enskog method, it can be proved that a first-order approximation with respect to $\varepsilon$ of (1) is given by the following differential drift-flux model :

$$
\left\{\begin{array}{l}
\frac{\partial \varrho}{\partial t}+\frac{\partial}{\partial x}(\varrho u)=0 \\
\frac{\partial}{\partial t}(\varrho Y)+\frac{\partial}{\partial x}\left(\varrho Y u+\varrho Y(1-Y) u_{r}\right)=0 \\
\frac{\partial}{\partial t}(\varrho u)+\frac{\partial}{\partial x}\left(\varrho u^{2}+p+\varrho Y(1-Y) u_{r}^{2}\right)=\varrho g \\
\frac{\partial}{\partial t}(\varrho e)+\frac{\partial}{\partial x}\left((\varrho e+p) u+\varrho Y(1-Y) u_{r}^{2} u\right)=\varrho g u
\end{array}\right.
$$


where the relative velocity $u_{r}$ is given by the differential drift law

$$
\left|u_{r}\right| u_{r}=\frac{\varrho Y(1-Y)}{\Lambda}\left(\frac{1}{\varrho_{1}}-\frac{1}{\varrho_{2}}\right) \frac{\partial p}{\partial x} .
$$

Closure relations for $\rho_{1}, \rho_{2}$ and $p$ are not detailed here and we refer again to [14], [24]. We note that (23) involves the drag-force coefficient but is independent of the pressure relaxation coefficient. It also depends on the unknown derivatives by the pressure gradient $\frac{\partial p}{\partial x}$. Of course, if we assume for instance that the pressure gradient is balanced by the gravity force, that is

$$
\frac{\partial p}{\partial x}=-\varrho g,
$$

as it is the case for permanent flows, (23) has to be replaced by the following algebraic (zeroth-order) drift law

$$
\left|u_{r}\right| u_{r}=-\frac{\varrho Y(1-Y)}{\Lambda}\left(\frac{1}{\varrho_{1}}-\frac{1}{\varrho_{2}}\right) \varrho g .
$$

We obtain in this case the classical drift-flux one-fluid model (see for instance [27]), which definitely proves a hierarchy between the drift-flux models and the seven-equation model.

This asymptotic property holds true at the continuum level. A challenging issue is to retain the validity of this asymptotic behavior from a numerical point of view, by means of an asymptotic-preserving scheme for the seven-equation model. In other words, such a scheme is expected to provide a consistent approximation of the drift-flux model $(22),(23)$ in the asymptotic regime $\varepsilon \rightarrow 0$.

The rest of the paper is precisely devoted to the derivation of a numerical scheme for the seven-equation model. It will be based on a splitting strategy between the pressure relaxation terms and the other ones, namely the convective terms and both the gravity and drag force terms. A particular attention will be paid to the latter terms for which we will propose an approximate Godunov method. In particular, we emphasize from now on that the sources will be included in the definition of the underlying approximate Riemann solver, and thus will receive an upwind treatment. The motivation to split the pressure relaxation terms comes from the very fast pressure relaxation time-scales to the equilibrium $p_{1}=p_{2}$ used in (19).

\section{The principle of the numerical method}

As already mentioned, we will use a splitting strategy to solve numerically the system (1). Let us first introduce a space step $\Delta x$ and a time step $\Delta t$, and set

$$
x_{i}=i \Delta x, i \in \mathbb{Z}, \quad t^{n}=n \Delta t, n \in \mathbb{N} .
$$

We denote by $\mathbf{U}_{i}^{n}$ an approximation of $\mathbf{U}\left(x_{i}, t_{n}\right)$. Then, starting from $\mathbf{U}^{n}=$ $\left(\mathbf{U}_{i}^{n}\right)_{i \in \mathbb{Z}}$, we propose to compute the approximate solution $\mathbf{U}^{n+1}$ at time $t^{n+1}$ by means of the following two-step procedure. 
(i) First step. We solve numerically the system

$$
\left\{\begin{array}{l}
\frac{\partial \alpha_{k}}{\partial t}+u_{I} \frac{\partial \alpha_{k}}{\partial x}=0 \\
\frac{\partial}{\partial t}\left(\alpha_{k} \varrho_{k}\right)+\frac{\partial}{\partial x}\left(\alpha_{k} \varrho_{k} u_{k}\right)=0 \\
\frac{\partial}{\partial t}\left(\alpha_{k} \varrho_{k} u_{k}\right)+\frac{\partial}{\partial x}\left(\alpha_{k}\left(\varrho_{k} u_{k}^{2}+p_{k}\right)\right)-p_{I} \frac{\partial \alpha_{k}}{\partial x}=\alpha_{k} \varrho_{k} g-K\left(u_{k}-u_{l}\right) \\
\frac{\partial}{\partial t}\left(\alpha_{k} \varrho_{k} e_{k}\right)+\frac{\partial}{\partial x}\left(\alpha_{k}\left(\varrho_{k} e_{k}+p_{k}\right) u_{k}\right)-p_{I} u_{I} \frac{\partial \alpha_{k}}{\partial x}= \\
=\alpha_{k} \varrho_{k} g u_{k}-u_{I} K\left(u_{k}-u_{l}\right)
\end{array}\right.
$$

(obtained from (1) by setting $\Theta=0$ ) using $\mathbf{U}^{n}$ as initial condition. We denote by $\mathbf{U}^{n+1 / 2}$ the solution of (25) obtained at time $t^{n+1}$. This will be achieved by means of a new Godunov-type method described in the next section, and which is able to handle the source terms (the drag force and gravity) in a consistent way in order to preserve the asymptotic properties of (1). Such an approach, leading to an upwind treatment of the sources, seems indeed natural if we refer for instance to [10]. In this paper, the authors consider the classical gas dynamics system with gravity and friction, and propose an asymptotic-preserving Godunov-type scheme (when the friction coefficient goes to infinity) including the sources in the derivation of the underlying approximate Riemann solver. Here, in some sense, the seven-equation can be understood as two gas dynamics system with friction and gravity coupled by an additional equation on the void fractions, which justifies our approach.

(ii) Second step. The pressure relaxation term are now taken into account : the numerical solution of (1) at time $t^{n+1}$ is computed by solving the following ODE system

$$
\left\{\begin{array}{l}
\frac{d \alpha_{k}}{d t}=\Theta\left(p_{k}-p_{l}\right) \\
\frac{d}{d t}\left(\alpha_{k} \varrho_{k}\right)=\frac{d}{d t}\left(\alpha_{k} \varrho_{k} u_{k}\right)=0 \\
\alpha_{k} \varrho_{k} \frac{d e_{k}}{d t}=p_{I} \Theta\left(p_{k}-p_{l}\right)
\end{array}\right.
$$

or equivalently

$$
\left\{\begin{array}{l}
\frac{d \alpha_{k}}{d t}=\Theta\left(p_{k}-p_{l}\right), \\
\alpha_{k} \frac{d \varrho_{k}}{d t}=-\varrho_{k} \frac{d \alpha_{k}}{d t} \\
\alpha_{k} \varrho_{k} \frac{d u_{k}}{d t}=0 \\
\alpha_{k} \varrho_{k} \frac{d \varepsilon_{k}}{d t}=p_{I} \frac{d \alpha_{k}}{d t}
\end{array}\right.
$$


with the initial condition $\mathbf{U}^{n+1 / 2}$. Actually, this system has already been analyzed and solved numerically in [21]. For reader's convenience and for the sake of completeness, we briefly recall the main ideas of this strategy that we have used in practice, and refer the reader to [21] for the details. The main objective of the procedure is to preserve the positivity of the pressures $p_{1}$ and $p_{2}$ as well as the maximum principle $\alpha_{k} \in[0,1]$ for the void fractions.

Let us first focus on the void fractions. Using (10), the first equation of (26) equivalently writes

$$
\frac{1}{\alpha_{1} \alpha_{2}} \frac{d \alpha_{1}}{d t}=\frac{1}{\tau_{p}} \frac{p_{1}-p_{2}}{p_{1}+p_{2}}
$$

that is, since $\alpha_{1}+\alpha_{2}=1$,

$$
\frac{d}{d t} \ln \left(\frac{\alpha_{1}}{1-\alpha_{1}}\right)=\frac{1}{\tau_{p}} \frac{p_{1}-p_{2}}{p_{1}+p_{2}}
$$

We thus get after integration

$$
\left(\frac{\alpha_{1}}{1-\alpha_{1}}\right)(t)=\left(\frac{\alpha_{1}}{1-\alpha_{1}}\right)^{n+1 / 2} \exp \left(\frac{1}{\tau_{p}} \int_{0}^{t}\left(\frac{p_{1}-p_{2}}{p_{1}+p_{2}}\right)(\tau) d \tau\right) .
$$

This formula clearly provides us with an unique definition of $\alpha_{k}(t) \in[0,1]$ whatever be the proposed quadrature formula and provided that $\left(\alpha_{k}\right)^{n+1 / 2} \in[0,1]$, $k=1,2$.

Regarding the two pressures $p_{1}$ and $p_{2}$, we first write using (27)

$$
\frac{d p_{k}}{d t}=\frac{\partial p_{k}}{\partial \rho_{k}} \frac{d \rho_{k}}{d t}+\frac{\partial p_{k}}{\partial \varepsilon_{k}} \frac{d \varepsilon_{k}}{d t}=-\left(\frac{\partial p_{k}}{\partial \rho_{k}} \frac{\rho_{k}}{\alpha_{k}}-\frac{\partial p_{k}}{\partial \varepsilon_{k}} \frac{p_{I}}{\alpha_{k} \rho_{k}}\right) \frac{d \alpha_{k}}{d t}, \quad k=1,2 .
$$

To avoid cumbersome notations, we propose to set

$$
A_{k}=\left(\frac{\partial p_{k}}{\partial \rho_{k}} \frac{\rho_{k}}{\alpha_{k}}-\frac{\partial p_{k}}{\partial \varepsilon_{k}} \frac{p_{I}}{\alpha_{k} \rho_{k}}\right), \quad k=1,2 .
$$

Substracting these two equations and using the first equation of (26) leads to

$$
\frac{d}{d t}\left(p_{k}-p_{l}\right)+\Theta\left(A_{k}-A_{l}\right)\left(p_{k}-p_{l}\right)=0,
$$

which gives after integration

$$
\left(p_{k}-p_{l}\right)(t)=\left(p_{k}-p_{l}\right)^{n+1 / 2} \exp \left(-\int_{0}^{t} \Theta\left(A_{k}-A_{l}\right)(\tau) d \tau\right), \quad k=1,2 .
$$

This first equation proves that $\left(p_{k}-p_{l}\right)(t)$ and $\left(p_{k}-p_{l}\right)^{n+1 / 2}$ keep the same sign for all $t$. Now dividing (29) by $p_{k}$ gives

$$
\frac{d}{d t} \ln p_{k}+\frac{\alpha_{k} A_{k}}{p_{k}} \frac{d}{d t} \ln \alpha_{k}=0
$$

and then

$$
\frac{d}{d t} \ln p_{1} p_{2}+\sum_{k=1}^{2} \frac{\alpha_{k} A_{k}}{p_{k}} \frac{d}{d t} \ln \alpha_{k}=0
$$


Integrating gives

$$
\left(p_{1} p_{2}\right)(t)=\left(p_{1} p_{2}\right)^{n+1 / 2} \exp \left(-\int_{0}^{t}\left(\sum_{k=1}^{2} \frac{\alpha_{k} A_{k}}{p_{k}} \frac{d}{d t} \ln \alpha_{k}\right)(\tau) d \tau\right) .
$$

This second equation shows that $p_{1}$ and $p_{2}$ have necessarily the same sign provided that $\left(p_{1} p_{2}\right)^{n+1 / 2}>0$. Formulas (30) and (31) thus provide us with unique positive values of $p_{1}(t)$ and $p_{2}(t)$ for all time $t$, provided that $\left(p_{1}\right)^{n+1 / 2}>0$ and $\left(p_{2}\right)^{n+1 / 2}>0$.

On the ground of the above developments, the definition of the numerical solution to system (26) given in [21] is based on a natural discretization of

$$
\left\{\begin{array}{l}
\left(\frac{\alpha_{1}}{1-\alpha_{1}}\right)(t)=\left(\frac{\alpha_{1}}{1-\alpha_{1}}\right)^{n+1 / 2} \exp \left(\frac{1}{\tau_{p}} \int_{0}^{t}\left(\frac{p_{1}-p_{2}}{p_{1}+p_{2}}\right)(\tau) d \tau\right), \\
\left(\alpha_{k} \varrho_{k}\right)(t)=\left(\alpha_{k} \varrho_{k}\right)^{n+1 / 2}, \\
\left(\alpha_{k} \varrho_{k} u_{k}\right)(t)=\left(\alpha_{k} \varrho_{k} u_{k}\right)^{n+1 / 2}, \\
\left(p_{1}-p_{2}\right)(t)=\left(p_{1}-p_{2}\right)^{n+1 / 2} \exp \left(-\int_{0}^{t} \Theta\left(A_{1}-A_{2}\right)(\tau) d \tau\right), \\
\left(p_{1} p_{2}\right)(t)=\left(p_{1} p_{2}\right)^{n+1 / 2} \exp \left(-\int_{0}^{t}\left(\sum_{k=1}^{2} \frac{\Theta A_{k}}{p_{k}}\left(p_{k}-p_{l}\right)\right)(\tau) d \tau\right) .
\end{array}\right.
$$

This strategy clearly allows to define positive pressures $p_{k}$ and void fractions $\alpha_{k}$ lying in $[0,1]$. The partial densities also remain positive provided that they are at the end of the first step.

We now focus in the next section on the numerical approximation of the first step and more precisely on the derivation of a consistent approximate Riemann solver for (25).

\section{The approximate Riemann solver}

The system (25) is again of the general form (3) where $\mathbf{U}, \mathbf{F}(\mathbf{U})$ and $\mathbf{B}(\mathbf{U})$ are still given by (4),(5) and (8) respectively while $\mathbf{S}(\mathbf{U})$ is now defined by

$$
\mathbf{S}(\mathbf{U})=\left(\begin{array}{c}
0 \\
\mathbf{S}_{1}(\mathbf{U}) \\
\mathbf{S}_{2}(\mathbf{U})
\end{array}\right), \quad \mathbf{S}_{k}(\mathbf{U})=\left(\begin{array}{c}
0 \\
\alpha_{k} \varrho_{k} g-K\left(u_{k}-u_{l}\right) \\
\alpha_{k} \varrho_{k} g u_{k}-u_{I} K\left(u_{k}-u_{l}\right)
\end{array}\right) .
$$

Our purpose is now to construct an approximate Riemann solver for the system (25).

\subsection{Generalities}

We first recall in this section a fairly general method of construction of Riemann solvers for a nonconservative systems with sources of $p$ equations of the form 
(3). More precisely, our main objective is to recall, following Gallice [18], [19] and [20], the notion of consistency with the integral form of (25) of such an approximate Riemann solver.

We first introduce a simple Riemann solver of the form

$$
\mathbf{W}_{\Delta}\left(\frac{x}{t} ; \mathbf{U}_{L}, \mathbf{U}_{R}\right)=\left\{\begin{array}{l}
\mathbf{U}_{1}=\mathbf{U}_{L}, \quad \frac{x}{t}<\sigma_{1}, \\
\mathbf{U}_{k}, \quad \sigma_{k-1}<\frac{x}{t}<\sigma_{k}, \quad k=2, . ., m, \\
\mathbf{U}_{m+1}=\mathbf{U}_{R}, \quad \frac{x}{t}>\sigma_{m} .
\end{array}\right.
$$

It consists of $m$ waves with speeds $\sigma_{k}=\sigma_{k}\left(\mathbf{U}_{L}, \mathbf{U}_{R}\right), 1 \leq k \leq m$, and $m-1$ intermediate states $\mathbf{U}_{k}, 2 \leq k \leq m$, which may depend on $\Delta=(\Delta x, \Delta t)^{1}$. As it is natural, we will first require

$$
\lim _{\substack{\mathbf{U}_{L}, \mathbf{U}_{R} \rightarrow \mathbf{U} \\ \Delta \rightarrow 0}} \mathbf{W}_{\Delta}\left(\frac{x}{t} ; \mathbf{U}_{L}, \mathbf{U}_{R}\right)=\mathbf{U}
$$

Let us now define the consistency in the integral sense.

\subsubsection{Consistency with the integral form of (25)}

We first observe that, under the CFL condition

$$
\max _{1 \leq k \leq m}\left|\sigma_{k}\right| \frac{\Delta t}{\Delta x} \leq \frac{1}{2}
$$

we have

$$
\frac{1}{\Delta x} \int_{-\frac{\Delta x}{2}}^{\frac{\Delta x}{2}} \mathbf{W}_{\Delta}\left(\frac{x}{\Delta t} ; \mathbf{U}_{L}, \mathbf{U}_{R}\right) d x=\frac{1}{2}\left(\mathbf{U}_{L}+\mathbf{U}_{R}\right)-\frac{\Delta t}{\Delta x} \sum_{k=1}^{m} \sigma_{k}\left(\mathbf{U}_{k+1}-\mathbf{U}_{k}\right)
$$

so that

$$
\int_{0}^{\Delta t} \int_{-\frac{\Delta x}{2}}^{\frac{\Delta x}{2}} \frac{\partial}{\partial t} \mathbf{W}_{\Delta}\left(\frac{x}{t} ; \mathbf{U}_{L}, \mathbf{U}_{R}\right) d x d t=-\Delta t \sum_{k=1}^{m} \sigma_{k}\left(\mathbf{U}_{k+1}-\mathbf{U}_{k}\right) .
$$

Next, integrating (25) in space and time leads to

$\int_{0}^{\Delta t} \int_{-\frac{\Delta x}{2}}^{\frac{\Delta x}{2}} \frac{\partial}{\partial t} \mathbf{U} d x d t+\int_{0}^{\Delta t} \int_{-\frac{\Delta x}{2}}^{\frac{\Delta x}{2}}\left\{\frac{\partial}{\partial x} \mathbf{F}(\mathbf{U})+\mathbf{B}(\mathbf{U}) \frac{\partial \mathbf{U}}{\partial x}\right\} d x d t=\int_{0}^{\Delta t} \int_{-\frac{\Delta x}{2}}^{\frac{\Delta x}{2}} \mathbf{S}(\mathbf{U}) d x d t$

which gives, focusing ourselves on simple Riemann solutions of the form (34),

$\int_{-\frac{\Delta x}{2}}^{\frac{\Delta x}{2}} \mathbf{W}\left(\frac{x}{\Delta t} ; \mathbf{U}_{L}, \mathbf{U}_{R}\right) d x=\frac{\Delta x}{2}\left(\mathbf{U}_{L}+\mathbf{U}_{R}\right)-\int_{0}^{\Delta t} \int_{-\frac{\Delta x}{2}}^{\frac{\Delta x}{2}}\left\{\frac{\partial}{\partial x} \mathbf{F}(\mathbf{W})+\mathbf{B}(\mathbf{W}) \frac{\partial \mathbf{W}}{\partial x}-\mathbf{S}(\mathbf{W})\right\} d x d t$.

\footnotetext{
${ }^{1}$ In the sequel, the subscript $\Delta=(\Delta x, \Delta t)$ will indicate a dependence on $\Delta x$ and $\Delta t$.
} 
Consistency in the integral sense consists in imposing

$$
\int_{0}^{\Delta t} \int_{-\frac{\Delta x}{2}}^{\frac{\Delta x}{2}}\left\{\frac{\partial}{\partial x} \mathbf{F}(\mathbf{W})+\mathbf{B}(\mathbf{W}) \frac{\partial \mathbf{W}}{\partial x}-\mathbf{S}(\mathbf{W})\right\} d x d t \approx \Delta t \sum_{k=1}^{m} \sigma_{k}\left(\mathbf{U}_{k+1}-\mathbf{U}_{k}\right) .
$$

More precisely, the Riemann solver (34) is said to be consistent with (3) if there exists a $p \times p$ matrix $\mathbf{B}_{\Delta}\left(\mathbf{U}_{L}, \mathbf{U}_{R}\right)$ and a vector $\mathbf{S}_{\Delta}\left(\mathbf{U}_{L}, \mathbf{U}_{R}\right) \in \mathbb{R}^{p}$ with

$$
\lim _{\substack{\mathbf{U}_{L}, \mathbf{U}_{R} \rightarrow \mathbf{U} \\ \Delta \rightarrow 0}} \mathbf{B}_{\Delta}\left(\mathbf{U}_{L}, \mathbf{U}_{R}\right)=\mathbf{B}(\mathbf{U})
$$

and

$$
\lim _{\substack{\mathbf{U}_{L}, \mathbf{U}_{R} \rightarrow \mathbf{U} \\ \Delta \rightarrow 0}} \mathbf{S}_{\Delta}\left(\mathbf{U}_{L}, \mathbf{U}_{R}\right)=\mathbf{S}(\mathbf{U})
$$

such that under the CFL condition (36)

$$
\Delta \mathbf{F}+\mathbf{B}_{\Delta}\left(\mathbf{U}_{L}, \mathbf{U}_{R}\right) \Delta \mathbf{U}-\Delta x \mathbf{S}_{\Delta}\left(\mathbf{U}_{L}, \mathbf{U}_{R}\right)=\sum_{k=1}^{m} \sigma_{k}\left(\mathbf{U}_{k+1}-\mathbf{U}_{k}\right)
$$

where as usual $\Delta \mathbf{U}=\mathbf{U}_{L}-\mathbf{U}_{R}$ and $\Delta \mathbf{F}=\mathbf{F}\left(\mathbf{U}_{R}\right)-\mathbf{F}\left(\mathbf{U}_{L}\right)$. Note that the left-hand side of (36) is obtained by an exact calculation of the left-hand side of (37) where the exact matrix $\mathbf{B}$ and the exact vector $\mathbf{S}$ have been replaced with $\mathbf{B}_{\Delta}$ and $\mathbf{S}_{\Delta}$. Observe also that we recover the usual definition of consistency associated with systems of conservation laws when the non-conservative products and the sources are not present in the model (see for instance [26]).

\subsubsection{The Godunov-type method}

The Godunov-type method associated with the simple Riemann solver (34) is then defined as usual by

$$
\left\{\begin{aligned}
\mathbf{U}_{i}^{n+1}=\frac{1}{\Delta x}\left\{\int_{-\frac{\Delta x}{2}}^{0}\right. & \mathbf{W}_{\Delta}\left(\frac{x}{\Delta t} ; \mathbf{U}_{i}^{n}, \mathbf{U}_{i+1}^{n}\right) d x+ \\
& \left.+\int_{0}^{\frac{\Delta x}{2}} \mathbf{W}_{\Delta}\left(\frac{x}{\Delta t} ; \mathbf{U}_{i-1}^{n}, \mathbf{U}_{i}^{n}\right) d x\right\} .
\end{aligned}\right.
$$

Assuming that the Riemann solver (34) is consistent with (3) and setting

$$
\mathbf{G}_{\Delta}\left(\mathbf{U}_{L}, \mathbf{U}_{R}\right)=\frac{1}{2}\left\{\mathbf{F}\left(\mathbf{U}_{L}\right)+\mathbf{F}\left(\mathbf{U}_{R}\right)-\sum_{k=1}^{m}\left|\sigma_{k}\right|\left(\mathbf{U}_{k+1}-\mathbf{U}_{k}\right)\right\}
$$

one can check that, under the CFL condition (36), the associated Godunov-type method reads

$$
\left\{\begin{aligned}
\mathbf{U}_{i}^{n+1}=\mathbf{U}_{i}^{n}- & \frac{\Delta t}{\Delta x}\left(\mathbf{G}_{i+\frac{1}{2}}^{n}-\mathbf{G}_{i-\frac{1}{2}}^{n}\right)-\frac{\Delta t}{2 \Delta x}\left\{\mathbf{B}_{i-\frac{1}{2}}^{n}\left(\mathbf{U}_{i}^{n}-\mathbf{U}_{i-1}^{n}\right)+\right. \\
& \left.+\mathbf{B}_{i+\frac{1}{2}}^{n}\left(\mathbf{U}_{i+1}^{n}-\mathbf{U}_{i}^{n}\right)\right\}+\frac{\Delta t}{2}\left(\mathbf{S}_{i+\frac{1}{2}}^{n}+\mathbf{S}_{i-\frac{1}{2}}^{n}\right)
\end{aligned}\right.
$$


where for all i

$$
\left\{\begin{array}{c}
\mathbf{G}_{i+\frac{1}{2}}^{n}=\mathbf{G}_{\Delta}\left(\mathbf{U}_{i}^{n}, \mathbf{U}_{i+1}^{n}\right), \mathbf{B}_{i+\frac{1}{2}}^{n}=\mathbf{B}_{\Delta}\left(\mathbf{U}_{i}^{n}, \mathbf{U}_{i+1}^{n}\right), \\
\mathbf{S}_{i+\frac{1}{2}}^{n}=\mathbf{S}_{\Delta}\left(\mathbf{U}_{i}^{n}, \mathbf{U}_{i+1}^{n}\right) .
\end{array}\right.
$$

Once the approximate Riemann solver is defined according to (40), the proposed Godunov-type method is then very classical. We note however that the nonconservative products and the sources are taken into account in the definition of consistency, and then clearly receive an upwind treatment in formula (43).

\subsection{Construction of the Riemann solver}

In order to construct an approximate Riemann solver for the system (25), it is first convenient to associate to (25) the linearized system

$$
\left\{\begin{array}{l}
\frac{\partial \alpha_{k}}{\partial t}+u_{I} \frac{\partial \alpha_{k}}{\partial x}=0 \\
\frac{\partial \alpha_{k} \varrho_{k}}{\partial t}+\frac{\partial \alpha_{k} \varrho_{k} u_{k}}{\partial x}=0 \\
\frac{\partial \alpha_{k} \varrho_{k} u_{k}}{\partial t}+\frac{\partial}{\partial x}\left(\alpha_{k} \varrho_{k} u_{k}^{2}+\Pi_{k}\right)-p_{I} \frac{\partial \alpha_{k}}{\partial x}=\alpha_{k} \varrho_{k} g-K\left(u_{k}-u_{l}\right) \\
\frac{\partial \alpha_{k} \varrho_{k} e_{k}}{\partial t}+\frac{\partial}{\partial x}\left(\left(\alpha_{k} \varrho_{k} e_{k}+\Pi_{k}\right) u_{k}\right)-p_{I} u_{I} \frac{\partial \alpha_{k}}{\partial x}= \\
=\alpha_{k} \varrho_{k} g u_{k}-u_{I} K\left(u_{k}-u_{l}\right) \\
\frac{\partial C_{k}}{\partial t}+u_{I} \frac{\partial C_{k}}{\partial x}=0,
\end{array}\right.
$$

$k, l=1,2, l \neq k$, which is obtained by replacing $\alpha_{k} p_{k}$ by $\Pi_{k}$ and introducing a new variable $C_{k}>0$ which plays the role of a Lagrangian sound speed. This approach allows to relax the possibly strong nonlinearities involved in the pressure laws and is similar to the one used fo instance in the well-known HLLC approach, or in the relaxation schemes (see for instance [7] and [11], and the references therein). Setting

$$
\mathbf{V}_{k}=\left(\alpha_{k}, C_{k}, \varrho_{k}, u_{k}, e_{k}, \Pi_{k}\right)^{T}, \quad k=1,2,
$$

we assume as given the pair $\left(\mathbf{V}_{k, L}, \mathbf{V}_{k, R}\right)$ with

$$
\Pi_{k, \lambda}=\alpha_{k, \lambda} p_{k, \lambda}, \quad p_{k, \lambda}=p_{k}\left(\varrho_{k, \lambda}, \varepsilon_{k, \lambda}\right), \quad \lambda=L, R .
$$

Defining $\Pi_{k}$ at its equilibrium value $\alpha_{k} p_{k}$ for the left and right states of the Riemann initial condition implies that the consistency relations (40) are identical, the system under consideration being linearized (i.e. (45)) or not (i.e. (25)). We construct the Riemann solver as follows. 


\subsubsection{First notations and form of the approximate Riemann solver}

We first introduce an approximation

$$
u_{I}^{*}=u_{I}^{*}\left(\mathbf{V}_{L}, \mathbf{V}_{R}\right), \mathbf{V}_{\lambda}=\left(\begin{array}{c}
\mathbf{V}_{1, \lambda} \\
\mathbf{V}_{2, \lambda}
\end{array}\right), \lambda=L, R,
$$

of the interfacial velocity $u_{I}$ which will be determined in the sequel. Then the first and last equations (45) lead us to take

$$
\alpha_{k, \Delta}\left(\frac{x}{t} ; \mathbf{V}_{L}, \mathbf{V}_{R}\right)=\left\{\begin{array}{cc}
\alpha_{k, L}, & \frac{x}{t}<u_{I}^{*} \\
\alpha_{k, R}, & \frac{x}{t}>u_{I}^{*}
\end{array}\right.
$$

and

$$
C_{k, \Delta}\left(\frac{x}{t} ; \mathbf{V}_{L}, \mathbf{V}_{R}\right)= \begin{cases}C_{k, L}, & \frac{x}{t}<u_{I}^{*} \\ C_{k, R}, & \frac{x}{t}>u_{I}^{*} .\end{cases}
$$

For $k=1,2$, we next introduce a function $\mathbf{V}_{k, \Delta}$ of the form

$$
\mathbf{V}_{k, \Delta}\left(\frac{x}{t} ; \mathbf{V}_{L}, \mathbf{V}_{R}\right)= \begin{cases}\mathbf{V}_{k, L}, & \frac{x}{t}<\sigma_{k, 1}, \\ \mathbf{V}_{k, L}^{*}, & \sigma_{k, 1}<\frac{x}{t}<\sigma_{k, 2}, \\ \mathbf{V}_{k, R}^{*}, & \sigma_{k, 2}<\frac{x}{t}<\sigma_{k, 3}, \\ \mathbf{V}_{k, R}, & \frac{x}{t}>\sigma_{k, 3}\end{cases}
$$

where the wave speed estimates $\sigma_{k, 1}<\sigma_{k, 2}<\sigma_{k, 3}$ are given by

$$
\left\{\begin{array}{l}
\sigma_{k, 1}=u_{k, L}-\frac{C_{k, L}}{\varrho_{k, L}}, \\
\sigma_{k, 2}=\sigma_{2}=u_{I}^{*} \\
\sigma_{k, 3}=u_{k, R}+\frac{C_{k, R}}{\varrho_{k, R}} .
\end{array}\right.
$$

Note that by (48) and (49) we have

$$
\alpha_{k, \lambda}^{*}=\alpha_{k, \lambda}, \quad C_{k, \lambda}^{*}=C_{k, \lambda}, \quad \lambda=L, R .
$$

Now, if we set using obvious notations

$$
\mathbf{U}_{k, \lambda}=\mathbf{U}_{k}\left(\mathbf{V}_{k, \lambda}\right), \quad \mathbf{U}_{k, \lambda}^{*}=\mathbf{U}_{k}\left(\mathbf{V}_{k, \lambda}^{*}\right), \quad \mathbf{U}_{\lambda}=\left(\begin{array}{c}
\alpha_{1, \lambda} \\
\mathbf{U}_{1, \lambda} \\
\mathbf{U}_{2, \lambda}
\end{array}\right), \quad \lambda=L, R,
$$


the Riemann solver is taken in the form

$$
\mathbf{W}_{\Delta}\left(\frac{x}{t} ; \mathbf{U}_{L}, \mathbf{U}_{R}\right)=\left(\begin{array}{c}
\alpha_{1, \Delta}\left(\frac{x}{t} ; \mathbf{V}_{L}, \mathbf{V}_{R}\right) \\
\mathbf{W}_{1, \Delta}\left(\frac{x}{t} ; \mathbf{U}_{L}, \mathbf{U}_{R}\right) \\
\mathbf{W}_{2, \Delta}\left(\frac{x}{t} ; \mathbf{U}_{L}, \mathbf{U}_{R}\right)
\end{array}\right)
$$

with

$$
\mathbf{W}_{k, \Delta}\left(\frac{x}{t} ; \mathbf{U}_{L}, \mathbf{U}_{R}\right)= \begin{cases}\mathbf{U}_{k, L}, & \frac{x}{t}<\sigma_{k, 1}, \\ \mathbf{U}_{k, L}^{*}, & \sigma_{k, 1}<\frac{x}{t}<\sigma_{2}, \\ \mathbf{U}_{k, R}^{*}, & \sigma_{2}<\frac{x}{t}<\sigma_{k, 3}, \\ \mathbf{U}_{k, R}, & \frac{x}{t}>\sigma_{k, 3} .\end{cases}
$$

Indeed, in (50) (resp. in (55)), $\mathbf{V}_{k, \lambda}^{*}$ (resp. $\mathbf{U}_{k, \lambda}^{*}$ ) and $\sigma_{2}=u_{I}^{*}$ will depend on $\Delta=(\Delta x, \Delta t)$.

We note that the unknowns $\mathbf{V}_{k, \lambda}$ or $\mathbf{U}_{k, \lambda}$ associated with the phase $k$ possibly jump across the $k$-acoustic waves $\sigma_{k, 1}$ and $\sigma_{k, 3}$ and the coupling wave $\sigma_{2}$, but never across the $l$-acoustic waves $\sigma_{l, 1}$ and $\sigma_{l, 3}, l \neq k$. This is natural since $\alpha_{k}$ is constant across all the acoustic waves by (48), so that the evolution of the two phases are decoupled in these regions (see section 2.1 above). We also note that the contact-discontinuities $u_{k}, k=1,2$ have been removed from the Riemann solver like in a HLL-approach. This allows at least in the subsonic regime to drop the difficulty related to the wave positions, that is to avoid switching from one wave configuration to another depending on whether $u_{1}<u_{2}$ or not. We will then get an explicit approximate Riemann solver.

\subsubsection{The consistency relations}

It thus remains to define the wave speed $u_{I}^{*}$ and the intermediate states $\mathbf{V}_{k, \lambda}^{*}, k=$ $1,2, \lambda=L, R$, in such a way that the approximate Riemann solver is consistent with the integral form of system (25), or equivalently that the consistency relations (40) are valid. This first necessitates to define the matrix $\mathbf{B}_{\Delta}$ and the vector $\mathbf{S}_{\Delta}$. We propose to simply set

$$
\mathbf{S}_{\Delta}=\left(\begin{array}{c}
0 \\
\mathbf{S}_{1, \Delta} \\
\mathbf{S}_{2, \Delta}
\end{array}\right), \quad \mathbf{S}_{k, \Delta}=\left(\begin{array}{c}
0 \\
\widetilde{\alpha_{k} \varrho_{k}} g-K \widetilde{\left(u_{k}-u_{l}\right)} \\
\widetilde{\alpha_{k} \varrho_{k} g \tilde{u}_{k}}-\tilde{u}_{I} K \widetilde{\left(u_{k}-u_{l}\right)}
\end{array}\right)
$$


and

$$
\mathbf{B}_{\Delta}=\left(\begin{array}{cc}
\tilde{u}_{I} & \vdots \\
0 & \vdots \\
-\tilde{p}_{I} & \vdots \\
-\tilde{p}_{I} \tilde{u}_{I} & \vdots \\
0 & \vdots \\
\tilde{p}_{I} & \vdots \\
\tilde{p}_{I} \tilde{u}_{I} & \vdots
\end{array}\right)
$$

where $\widetilde{\alpha_{k} \varrho_{k}}, \tilde{u}_{k}, K \widetilde{\left(u_{k}-u_{l}\right)}, \tilde{u}_{I}$ and $\tilde{p}_{I}$ are consistent approximations of $\alpha_{k} \varrho_{k}, u_{k}, K\left(u_{k}-\right.$ $\left.u_{l}\right), u_{I}$ and $p_{I}$ respectively. In addition, we assume

$$
K\left(\widetilde{u_{2}-u_{1}}\right)=-K\left(\widetilde{u_{1}-u_{2}}\right) .
$$

Then the general consistency relation (40) gives on the one hand

$$
\tilde{u}_{I}=u_{I}^{*}
$$

and on the other hand for $k=1,2$

$$
\left\{\begin{array}{l}
\Delta \mathbf{F}_{k}-\left(\begin{array}{c}
0 \\
\tilde{p}_{I} \\
\tilde{p}_{I} \tilde{u}_{I}
\end{array}\right) \Delta \alpha_{k}-\Delta x \mathbf{S}_{k, \Delta}=\sigma_{k, 1}\left(\mathbf{U}_{k, L}^{*}-\mathbf{U}_{k, L}\right)+ \\
+u_{I}^{*}\left(\mathbf{U}_{k, R}^{*}-\mathbf{U}_{k, L}^{*}\right)+\sigma_{k, 3}\left(\mathbf{U}_{k, R}-\mathbf{U}_{k, R}^{*}\right) .
\end{array}\right.
$$

\subsubsection{Definition of the intermediate states}

We have now to determine the pair $\left(\mathbf{V}_{k, L}^{*}, \mathbf{V}_{k, R}^{*}\right)$ or equivalently the pair $\left(\mathbf{U}_{k, L}^{*}, \mathbf{U}_{k, R}^{*}\right)$.

The mass equations. We begin by writing the Rankine-Hugoniot jump relations for the mass conservation equation at each wave with speed $\sigma_{k, l}, 1 \leq l \leq 3$ :

$$
\left\{\begin{array}{l}
\sigma_{k, 1}\left(\varrho_{k, L}^{*}-\varrho_{k, L}\right)=\varrho_{k, L}^{*} u_{k, L}^{*}-\varrho_{k, L} u_{k, L}, \\
\sigma_{2}\left(\alpha_{k, R} \varrho_{k, R}^{*}-\alpha_{k, L} \varrho_{k, L}^{*}\right)=\alpha_{k, R} \varrho_{k, R}^{*} u_{k, R}^{*}-\alpha_{k, L} \varrho_{k, L}^{*} u_{k, L}^{*} \\
\sigma_{k, 3}\left(\varrho_{k, R}-\varrho_{k, R}^{*}\right)=\varrho_{k, R} u_{k, R}-\varrho_{k, R}^{*} u_{k, R}^{*}
\end{array}\right.
$$

By summing the above equations (59), we obtain that the first component of the consistency condition (58) is trivially satisfied. Together with the expressions (51) of $\sigma_{k, l}, 1 \leq l \leq 3$ the first and third relations (59) yield

$$
\left\{\begin{array}{c}
\sigma_{k, 1}=u_{k, L}-\frac{C_{k, L}}{\varrho_{k, L}}=u_{k, L}^{*}-\frac{C_{k, L}}{\varrho_{k, L}^{*}}, \\
\sigma_{k, 3}=u_{k, R}+\frac{C_{k, R}}{\varrho_{k, R}}=u_{k, R}^{*}+\frac{C_{k, R}}{\varrho_{k, R}^{*}}
\end{array}\right.
$$


while the second relation (59) reads

$$
j_{k} \stackrel{\text { def }}{=} \alpha_{k, L} \varrho_{k, L}^{*}\left(u_{k, L}^{*}-u_{I}^{*}\right)=\alpha_{k, R} \varrho_{k, R}^{*}\left(u_{k, R}^{*}-u_{I}^{*}\right) .
$$

The momentum equations. We next consider the momentum conservation equation. The idea consists here in taking into account the source term only when crossing the wave with speed $\sigma_{2}=u_{I}^{*}$. We thus write the RankineHugoniot jump relations for the homogeneous momentum conservation equation at the acoustic waves whose speeds are $\sigma_{k, 1}$ and $\sigma_{k, 3}$ :

$$
\left\{\begin{aligned}
\sigma_{k, 1} \alpha_{k, L}\left(\varrho_{k, L}^{*} u_{k, L}^{*}-\varrho_{k, L} u_{k, L}\right)= & \alpha_{k, L}\left(\varrho_{k, L}^{*} u_{k, L}^{* 2}-\varrho_{k, L} u_{k, L}^{2}\right)+ \\
& +\Pi_{k, L}^{*}-\Pi_{k, L}, \\
\sigma_{k, 3} \alpha_{k, R}\left(\varrho_{k, R} u_{k, R}-\varrho_{k, R}^{*} u_{k, R}^{*}\right)= & \alpha_{k, R}\left(\varrho_{k, R} u_{k, R}^{2}-\varrho_{k, R}^{*} u_{k, R}^{* 2}\right)+ \\
& +\Pi_{k, R}-\Pi_{k, R}^{*} .
\end{aligned}\right.
$$

We supplement the above equations (62) with the second component of the consistency relation (58) which reads

$$
\left\{\begin{array}{l}
\left.\Delta\left(\alpha_{k} \varrho_{k} u_{k}^{2}+\Pi_{k}\right)-\tilde{p}_{I} \Delta \alpha_{k}-\Delta x\left(\widetilde{\alpha_{k} \varrho_{k}} g-K \widetilde{\left(u_{k}-u_{l}\right.}\right)\right)= \\
=\sigma_{k, 1} \alpha_{k, L}\left(\varrho_{k, L}^{*} u_{k, L}^{*}-\varrho_{k, L} u_{k, L}\right)+u_{I}^{*}\left(\alpha_{k, R} \varrho_{k, R}^{*} u_{k, R}^{*}-\alpha_{k, L} \varrho_{k, L}^{*} u_{k, L}^{*}\right)+ \\
\quad+\sigma_{k, 3} \alpha_{k, R}\left(\varrho_{k, R} u_{k, R}-\varrho_{k, R}^{*} u_{k, R}^{*}\right) .
\end{array}\right.
$$

Using (62), we obtain

$$
\left\{\begin{array}{l}
u_{I}^{*}\left(\alpha_{k, R} \varrho_{k, R}^{*} u_{k, R}^{*}-\alpha_{k, L} \varrho_{k, L}^{*} u_{k, L}^{*}\right)=\alpha_{k, R} \varrho_{k, R}^{*} u_{k, R}^{* 2}-\alpha_{k, L} \varrho_{k, L}^{*} u_{k, L}^{* 2}- \\
\left.-\tilde{p}_{I}\left(\alpha_{k, R}-\alpha_{k, L}\right)+\Pi_{k, R}^{*}-\Pi_{k, L}^{*}-\Delta x\left(\widetilde{\alpha_{k} \varrho_{k}} g-K \widetilde{\left(u_{k}-\right.} u_{l}\right)\right)
\end{array}\right.
$$

In fact, this equation may be viewed as a Rankine-Hugoniot jump relation when crossing the coupling wave whose speed is $u_{I}^{*}$ provided that the source term acts as a Dirac mass located along that wave.

Let us now arrange these relations in a more convenient way. By using the expression (60) of $\sigma_{k, 1}$ the first equation (62) becomes

$$
\left\{\begin{array}{r}
\left(u_{k, L}^{*}-\frac{C_{k, L}}{\varrho_{k, L}^{*}}\right) \varrho_{k, L}^{*} u_{k, L}^{*}-\left(u_{k, L}-\frac{C_{k, L}}{\varrho_{k, L}}\right) \varrho_{k, L} u_{k, L}= \\
=\varrho_{k, L}^{*} u_{k, L}^{* 2}-\varrho_{k, L} u_{k, L}^{2}+\frac{1}{\alpha_{k, L}}\left(\Pi_{k, L}^{*}-\Pi_{k, L}\right)
\end{array}\right.
$$

or

$$
\Pi_{k, L}^{*}=\Pi_{k, L}-\alpha_{k, L} C_{k, L}\left(u_{k, L}^{*}-u_{k, L}\right) .
$$

Similarly, the second equation (62) reads

$$
\Pi_{k, R}^{*}=\Pi_{k, R}+\alpha_{k, R} C_{k, R}\left(u_{k, R}^{*}-u_{k, R}\right) .
$$


On the other hand, by using (61), the equation (63) becomes

$$
\left\{\begin{aligned}
\Pi_{k, R}^{*}- & \Pi_{k, L}^{*}=j_{k}\left(u_{k, L}^{*}-u_{k, R}^{*}\right)+\tilde{p}_{I}\left(\alpha_{k, R}-\alpha_{k, L}\right)+ \\
& +\Delta x\left(\widetilde{\alpha_{k} \varrho_{k}} g-K\left(\widetilde{u_{k}-u_{l}}\right)\right) .
\end{aligned}\right.
$$

Then, replacing in (66) $\Pi_{k, L}^{*}$ and $\Pi_{k, R}^{*}$ by their expressions (64) and (65) gives

$$
\left\{\begin{array}{l}
\Delta \Pi_{k}-\tilde{p}_{I} \Delta \alpha_{k}=j_{k}\left(u_{k, L}^{*}-u_{k, R}^{*}\right)+2\left(\alpha_{k} C_{k} u_{k}\right)_{a}- \\
-\left(\alpha_{k, L} C_{k, L} u_{k, L}^{*}+\alpha_{k, R} C_{k, R} u_{k, R}^{*}\right)+\Delta x\left(\widetilde{\alpha_{k} \varrho_{k}} g-K\left(\widetilde{u_{k}-u_{l}}\right)\right)
\end{array}\right.
$$

where here and in all the sequel $\varphi_{a}=\frac{1}{2}\left(\varphi_{L}+\varphi_{R}\right)$ will denote the arithmetic average of any pair of quantities $\left(\varphi_{L}, \varphi_{R}\right)$. Notice that $(60)$ and (61) yield

$$
\left\{\begin{array}{c}
j_{k} u_{k, L}^{*}-\alpha_{k, L} C_{k, L} u_{k, L}^{*}=j_{k}\left(u_{k, L}^{*}-\frac{C_{k, L}}{\varrho_{k, L}^{*}}\right)-\alpha_{k, L} C_{k, L} u_{I}^{*}= \\
=\sigma_{k, 1} j_{k}-\alpha_{k, L} C_{k, L} u_{I}^{*}
\end{array}\right.
$$

and

$$
\left\{\begin{array}{c}
j_{k} u_{k, R}^{*}+\alpha_{k, R} C_{k, R} u_{k, R}^{*}=j_{k}\left(u_{k, R}^{*}+\frac{C_{k, R}}{\varrho_{k, R}^{*}}\right)+\alpha_{k, R} C_{k, R} u_{I}^{*}= \\
=\sigma_{k, 3} j_{k}+\alpha_{k, R} C_{k, R} u_{I}^{*} .
\end{array}\right.
$$

Then, taking (47) into account, we obtain

$$
\left\{\begin{array}{c}
\Delta\left(\alpha_{k} p_{k}\right)-\tilde{p}_{I} \Delta \alpha_{k}=\left(\sigma_{k, 1}-\sigma_{k, 3}\right) j_{k}+2\left\{\left(\alpha_{k} C_{k} u_{k}\right)_{a}-\left(\alpha_{k} C_{k}\right)_{a} u_{I}^{*}\right\} \\
+\Delta x\left(\widetilde{\alpha_{k} \varrho_{k}} g-K\left(\widetilde{u_{k}-u_{l}}\right)\right) .
\end{array}\right.
$$

The interfacial velocity $u_{I}^{*}$. Let us now define the approximate interfacial velocity $u_{I}^{*}$. The definition (12) of $u_{I}$ leads us to set

$$
u_{I}^{*}=\beta_{L} u_{1, L}^{*}+\left(1-\beta_{L}\right) u_{2, L}^{*}, \quad \beta_{L}=\frac{\chi \alpha_{1, L} \varrho_{1, L}^{*}}{\chi \alpha_{1, L} \varrho_{1, L}^{*}+(1-\chi) \alpha_{2, L} \varrho_{2, L}^{*}}
$$

or equivalently

$$
u_{I}^{*}=\beta_{R} u_{1, R}^{*}+\left(1-\beta_{R}\right) u_{2, R}^{*}, \quad \beta_{R}=\frac{\chi \alpha_{1, R} \varrho_{1, R}^{*}}{\chi \alpha_{1, R} \varrho_{1, R}^{*}+(1-\chi) \alpha_{2, R} \varrho_{2, R}^{*}} .
$$

This amounts to set

$$
\chi j_{1}+(1-\chi) j_{2}=0
$$

Calculation of $\left(\varrho_{k, L}^{*}, \varrho_{k, R}^{*}\right),\left(u_{k, L}^{*}, u_{k, R}^{*}\right),\left(\Pi_{k, L}^{*}, \Pi_{k, R}^{*}\right)$ for $k=1,2$, and $u_{I}^{*}$. We can then determine the pairs $\left(\varrho_{k, L}^{*}, \varrho_{k, R}^{*}\right),\left(u_{k, L}^{*}, u_{k, R}^{*}\right),\left(\Pi_{k, L}^{*}, \Pi_{k, R}^{*}\right)$. We 
first compute $j_{1}$ and $j_{2}$ in the following way. Summing the equations (67) for $k=1,2$, using $(56)$, we find

$$
\left\{\begin{aligned}
\Delta p= & \left(\sigma_{1,1}-\sigma_{1,3}\right) j_{1}+\left(\sigma_{2,1}-\sigma_{2,3}\right) j_{2}+2\left\{\left(\alpha_{1} C_{1} u_{1}\right)_{a}+\left(\alpha_{2} C_{2} u_{2}\right)_{a}-\right. \\
& \left.-\left(\left(\alpha_{1} C_{1}\right)_{a}+\left(\alpha_{2} C_{2}\right)_{a}\right) u_{I}^{*}\right\}+\Delta x\left(\widetilde{\alpha_{1} \varrho_{1}}+\widetilde{\alpha_{2} \varrho_{2}}\right) g .
\end{aligned}\right.
$$

In all the sequel, we will choose

$$
\widetilde{\alpha_{k} \varrho_{k}}=\left(\alpha_{k} \varrho_{k}\right)_{a}, \quad k=1,2 .
$$

Hence, we obtain

$$
\left\{\begin{aligned}
u_{I}^{*}= & \frac{1}{2\left(\left(\alpha_{1} C_{1}\right)_{a}+\left(\alpha_{2} C_{2}\right)_{a}\right)}\left\{\left(\sigma_{1,1}-\sigma_{1,3}\right) j_{1}+\left(\sigma_{2,1}-\sigma_{2,3}\right) j_{2}+\right. \\
& \left.+2\left(\left(\alpha_{1} C_{1} u_{1}\right)_{a}+\left(\alpha_{2} C_{2} u_{2}\right)_{a}\right)-\Delta p+\Delta x \varrho_{a} g\right\}
\end{aligned}\right.
$$

where the mean density $\varrho$ and the mean pressure $p$ are defined as in (20). Replacing $u_{I}^{*}$ by its expression (70) in the equation $(67)^{2}$ gives

$$
\left\{\begin{array}{c}
\left(\alpha_{2} C_{2}\right)_{a}\left(\sigma_{1,3}-\sigma_{1,1}\right) j_{1}-\left(\alpha_{1} C_{1}\right)_{a}\left(\sigma_{2,3}-\sigma_{2,1}\right) j_{2}- \\
-\Delta x\left(\left(\alpha_{1} C_{1}\right)_{a}+\left(\alpha_{2} C_{2}\right)_{a}\right) K\left(\widetilde{u_{2}-u_{1}}\right)= \\
=2\left\{\left(\alpha_{2} C_{2}\right)_{a}\left(\alpha_{1} C_{1} u_{1}\right)_{a}-\left(\alpha_{1} C_{1}\right)_{a}\left(\alpha_{2} C_{2} u_{2}\right)_{a}\right\}+ \\
+\left(\alpha_{1} C_{1}\right)_{a}\left(\Delta\left(\alpha_{2} p_{2}\right)-\tilde{p}_{I} \Delta \alpha_{2}\right)-\left(\alpha_{2} C_{2}\right)_{a}\left(\Delta\left(\alpha_{1} p_{1}\right)-\tilde{p}_{I} \Delta \alpha_{1}\right)+ \\
+\Delta x\left\{\left(\alpha_{2} C_{2}\right)_{a}\left(\alpha_{1} \varrho_{1}\right)_{a}-\left(\alpha_{1} C_{1}\right)_{a}\left(\alpha_{2} \varrho_{2}\right)_{a}\right\} g .
\end{array}\right.
$$

In order to determine $j_{1}$ and $j_{2}$ as solutions of the equations (68) and (71), we need now to specify the approximate drag term $K\left(\widetilde{\left.u_{2}-u_{1}\right)}\right.$. The simplest choice consists in setting

$$
K\left(\widetilde{u_{2}-u_{1}}\right)=\left(K\left(u_{2}-u_{1}\right)\right)_{a} .
$$

Assuming that $\tilde{p}_{I}$ does not depend on $j_{1}$ and $j_{2},(68)$ and (71) form a linear system in $\left(j_{1}, j_{2}\right)$ whose determinant

$$
-\chi\left(\alpha_{1} C_{1}\right)_{a}\left(\sigma_{2,3}-\sigma_{2,1}\right)-(1-\chi)\left(\alpha_{2} C_{2}\right)_{a}\left(\sigma_{1,3}-\sigma_{1,1}\right)
$$

never vanishes ${ }^{3}$.

Once $\left(j_{1}, j_{2}\right)$ is known, $u_{I}^{*}$ is given by $(70)$. Then, using (60) and (61), we obtain

$$
\left\{\begin{array}{l}
u_{k, L}^{*}=\frac{\left(C_{k, L}-\varrho_{k, L} u_{k, L}\right) j_{k}+\alpha_{k, L} C_{k, L} \varrho_{k, L} u_{I}^{*}}{\varrho_{k, L}\left(\alpha_{k, L} C_{k, L}-j_{k}\right)}, \\
u_{k, R}^{*}=\frac{\left(C_{k, R}+\varrho_{k, R} u_{k, R}\right) j_{k}+\alpha_{k, R} C_{k, R} \varrho_{k, R} u_{I}^{*}}{\varrho_{k, R}\left(\alpha_{k, R} C_{k, R}+j_{k}\right)}
\end{array}\right.
$$

${ }^{2}$ associated with the phase $k=1$ for instance.

${ }^{3}$ provided that the waves remain well ordered as they should, i.e., $\sigma_{k, 3}>\sigma_{k, 1}, k=1,2$. 
and

$$
\left\{\begin{array}{l}
\frac{1}{\varrho_{k, L}^{*}}=\frac{1}{\varrho_{k, L}}+\frac{u_{k, L}^{*}-u_{k, L}}{C_{k, L}}, \\
\frac{1}{\varrho_{k, R}^{*}}=\frac{1}{\varrho_{k, R}}-\frac{u_{k, R}^{*}-u_{k, R}}{C_{k, R}} .
\end{array}\right.
$$

The pair $\left(\Pi_{k, L}^{*}, \Pi_{k, R}^{*}\right)$ is then given by (64) and (65).

The energy equations and the calculation of $\left(e_{k, L}^{*}, e_{k, R}^{*}\right)$ for $k=1,2$. It remains to determine the pair $\left(e_{k, L}^{*}, e_{k, R}^{*}\right), k=1,2$, of intermediate values of the specific total energy by means of the energy conservation equation. First, the third component of the consistency relation (58) reads

$$
\left\{\begin{array}{c}
\Delta\left(\left(\alpha_{k} \varrho_{k} e_{k}+\Pi_{k}\right) u_{k}\right)-\tilde{p}_{I} \tilde{u}_{I} \Delta \alpha_{k}- \\
\left.-\Delta x\left\{\left(\alpha_{k} \varrho_{k}\right)_{a} g \tilde{u}_{k}-K \widetilde{\left(u_{k}-u_{l}\right.}\right) \tilde{u}_{I}\right\}= \\
=\sigma_{k, 1} \alpha_{k, L}\left(\varrho_{k, L}^{*} e_{k, L}^{*}-\varrho_{k, L} e_{k, L}\right)+ \\
+u_{I}^{*}\left(\alpha_{k, R} \varrho_{k, R}^{*} e_{k, R}^{*}-\alpha_{k, L} \varrho_{k, L}^{*} e_{k, L}^{*}\right)+\sigma_{k, 3} \alpha_{k, R}\left(\varrho_{k, R} e_{k, R}-\varrho_{k, R}^{*} e_{k, R}^{*}\right) .
\end{array}\right.
$$

Using again (60) and (61) together with (59), the consistency relation (74) becomes

$$
\left\{\begin{array}{l}
\left.\Delta\left(\Pi_{k} u_{k}\right)-\tilde{p}_{I} u_{I}^{*} \Delta \alpha_{k}-\Delta x\left\{\left(\alpha_{k} \varrho_{k}\right)_{a} g \tilde{u}_{k}-\tilde{u}_{I} K \widetilde{\left(u_{k}-u_{l}\right.}\right)\right\}= \\
=\left(j_{k}-\alpha_{k, L} C_{k, L}\right) e_{k, L}^{*}-\left(j_{k}+\alpha_{k, R} C_{k, R}\right) e_{k, R}^{*}+2\left(\alpha_{k} C_{k} e_{k}\right)_{a} .
\end{array}\right.
$$

where $\tilde{u}_{k}$ remains to be specified. This gives one equation for each phase.

We next need to derive another equation and a way to get it is as follows. As for the momentum equation we would like to write the Rankine-Hugoniot jump relations for the homogeneous energy conservation equation at the acoustic waves. This is excluded since we would obtain three conditions for two unknowns. Let us nevertheless write these jump relations where we have replaced $e_{k, l}^{*}$ and $e_{k, R}^{*}$ by $\bar{e}_{k, l}$ and $\bar{e}_{k, R}$ respectively: we find

$$
\left\{\begin{array}{c}
\sigma_{k, 1} \alpha_{k, L}\left(\varrho_{k, L}^{*} \bar{e}_{k, L}-\varrho_{k, L} e_{k, L}\right)=\alpha_{k, L}\left(\varrho_{k, L}^{*} \bar{e}_{k, L} u_{k, L}^{*}-\varrho_{k, L} e_{k, L} u_{k, L}\right)+ \\
+\Pi_{k, L}^{*} u_{k, L}^{*}-\Pi_{k, L} u_{k, L}
\end{array}\right.
$$

and

$$
\left\{\begin{array}{c}
\sigma_{k, 3} \alpha_{k, R}\left(\varrho_{k, R} e_{k, R}-\varrho_{k, R}^{*} \bar{e}_{k, R}\right)=\alpha_{k, R}\left(\varrho_{k, R} e_{k, R} u_{k, R}-\varrho_{k, R}^{*} \bar{e}_{k, R} u_{k, R}^{*}\right)+ \\
+\Pi_{k, R} u_{k, R}-\Pi_{k, R}^{*} u_{k, R}^{*} .
\end{array}\right.
$$

Using again (60), the above relations become

$$
\left\{\begin{array}{l}
\bar{e}_{k, L}=e_{k, L}+\frac{1}{\alpha_{k, L} C_{k, L}}\left(\Pi_{k, L} u_{k, L}-\Pi_{k, L}^{*} u_{k, L}^{*}\right), \\
\bar{e}_{k, R}=e_{k, R}+\frac{1}{\alpha_{k, R} C_{k, R}}\left(\Pi_{k, R}^{*} u_{k, R}^{*}-\Pi_{k, R} u_{k, R}\right) .
\end{array}\right.
$$


In order to determine $e_{k, L}^{*}$ and $e_{k, R}^{*}$, we solve an optimization problem. Since the consistency relation ( 75$)$ is of the form

$$
a_{1} e_{k, L}^{*}-a_{2} e_{k, R}^{*}=b
$$

with

$$
a_{1}=j_{k}-\alpha_{k, L} C_{k, L}, \quad a_{2}=j_{k}+\alpha_{k, R} C_{k, R}
$$

and

$$
\left\{\begin{aligned}
& b=\Delta\left(\Pi_{k} u_{k}\right)-\tilde{p}_{I} u_{I}^{*} \Delta \alpha_{k}-2\left(\alpha_{k} C_{k} e_{k}\right)_{a}- \\
&-\Delta x\left\{\left(\alpha_{k} \varrho_{k}\right)_{a} g \tilde{u}_{k}-u_{I}^{*} K\left(\widetilde{u_{k}-u_{l}}\right)\right\}
\end{aligned}\right.
$$

we minimize the quadratic functional

$$
J(x, y)=\left(x-\bar{e}_{k, L}\right)^{2}+\left(y-\bar{e}_{k, R}\right)^{2}
$$

under the linear constraint

$$
a_{1} x-a_{2} y=b_{1} .
$$

Clearly, this optimization problem has a unique solution $\left(x=e_{k, L}^{*}, y=e_{k, R}^{*}\right)$ which is easily determined

$$
\left\{\begin{array}{l}
e_{k, L}^{*}=\bar{e}_{k, L}+\frac{a_{1}}{a_{1}^{2}+a_{2}^{2}}\left(b-a_{1} \bar{e}_{k, L}+a_{2} \bar{e}_{k, R}\right), \\
e_{k, R}^{*}=\bar{e}_{k, R}-\frac{a_{2}}{a_{1}^{2}+a_{2}^{2}}\left(b-a_{1} \bar{e}_{k, L}+a_{2} \bar{e}_{k, R}\right) .
\end{array}\right.
$$

In addition, using (76)-(77), one can check that

$$
\left\{\begin{array}{c}
b-a_{1} \bar{e}_{k, L}+a_{2} \bar{e}_{k, R}=j_{k}\left(\bar{e}_{k, R}-\bar{e}_{k, L}\right)+\Pi_{k, R}^{*} u_{k, R}^{*}-\Pi_{k, L}^{*} u_{k, L}^{*}- \\
\left.-\tilde{p}_{I} u_{I}^{*} \Delta \alpha_{k}-\Delta x\left\{\left(\alpha_{k} \varrho_{k}\right)_{a} g \tilde{u}_{k}-u_{I}^{*} K \widetilde{\left(u_{k}-u_{l}\right.}\right)\right\}
\end{array}\right.
$$

The Riemann solver is therefore completely defined provided that we choose consistent approximations $\tilde{u}_{k}, \tilde{p}_{I}$ of $u_{k}$ and $p_{I}$. Many choices are of course possible. In practice, we first chose

$$
\tilde{u}_{k}=\frac{1}{2}\left(u_{k, L}^{*}+u_{k, R}^{*}\right)
$$

and then, in compliance with (12), (13) and (15),

$$
\tilde{p}_{I}=\tilde{\mu}\left(p_{1}\right)_{a}+(1-\tilde{\mu})\left(p_{2}\right)_{a}
$$

with

$$
\tilde{\mu}=(1-\chi)\left(\alpha_{2} \rho_{2} T_{2}\right)_{a}
$$

\section{The numerical scheme}

Let us now give an explicit form of the numerical scheme of solution of the system (1). 


\subsection{The first step: $\mathrm{U}^{n} \rightarrow \mathrm{U}^{n+1 / 2}$}

We first derive the explicit form of the Godunov-type scheme (43) associated with the Riemann solver introduced in the previous section. Using (42), it is a simple matter to check that the numerical flux $\mathbf{G}_{\Delta}\left(\mathbf{U}_{L}, \mathbf{U}_{R}\right)$ is given here by

$$
\mathbf{G}_{\Delta}\left(\mathbf{U}_{L}, \mathbf{U}_{R}\right)=\left(\begin{array}{c}
-\frac{1}{2}\left|u_{I}^{*}\right| \Delta \alpha_{1} \\
\mathbf{G}_{1, \Delta}\left(\mathbf{U}_{L}, \mathbf{U}_{R}\right) \\
\mathbf{G}_{2, \Delta}\left(\mathbf{U}_{L}, \mathbf{U}_{R}\right)
\end{array}\right)
$$

where

$$
\left\{\begin{array}{c}
\mathbf{G}_{k, \Delta}\left(\mathbf{U}_{L}, \mathbf{U}_{R}\right)=\frac{1}{2}\left\{\mathbf{F}_{k}\left(\mathbf{U}_{k, L}\right)+\mathbf{F}_{k}\left(\mathbf{U}_{k, R}\right)-\left|\sigma_{k, 1}\right|\left(\mathbf{U}_{k, L}^{*}-\mathbf{U}_{k, L}\right)-\right. \\
\left.-\left|u_{I}^{*}\right|\left(\mathbf{U}_{k, R}^{*}-\mathbf{U}_{k, L}^{*}\right)-\left|\sigma_{k, 3}\right|\left(\mathbf{U}_{k, R}-\mathbf{U}_{k, R}^{*}\right)\right\} .
\end{array}\right.
$$

Recall that $\left.\left.u_{I}^{*}=u_{I}^{*}\left(\mathbf{U}_{L}, \mathbf{U}_{R}\right), \tilde{p}_{I}=\tilde{p}_{I}\left(\mathbf{U}_{L}, \mathbf{U}_{R}\right), \widetilde{K\left(u_{k}-u_{l}\right.}\right)=\widetilde{K\left(u_{k}-u_{l}\right.}\right)\left(\mathbf{U}_{L}, \mathbf{U}_{R}\right)$ and $\tilde{u}_{k}=\tilde{u}_{k}\left(\mathbf{U}_{L}, \mathbf{U}_{R}\right)$ are consistent approximations of $u_{I}, p_{I}, K\left(u_{k}-u_{l}\right)$ and $u_{k}$ respectively. In all the sequel, we will set

$$
\left\{\begin{array}{l}
\left(u_{I}\right)_{i+1 / 2}^{n}=u_{I}^{*}\left(\mathbf{U}_{i}^{n}, \mathbf{U}_{i+1}^{n}\right),\left(p_{I}\right)_{i+1 / 2}^{n}=\tilde{p}_{I}\left(\mathbf{U}_{i}^{n}, \mathbf{U}_{i+1}^{n}\right) \\
\left(K\left(u_{k}-u_{l}\right)\right)_{i+1 / 2}^{n}=K\left(u_{k}-u_{l}\right)\left(\mathbf{U}_{i}^{n}, \mathbf{U}_{i+1}^{n}\right) \\
\left(u_{k}\right)_{i+1 / 2}^{n}=\tilde{u}_{k}\left(\mathbf{U}_{i}^{n}, \mathbf{U}_{i+1}^{n}\right)
\end{array}\right.
$$

Then, replacing $\mathbf{U}^{n+1}$ by $\mathbf{U}^{n+1 / 2}$, the first component of (43) reads here

$$
\left\{\begin{array}{c}
\left(\alpha_{1}\right)_{i}^{n+1 / 2}=\left(\alpha_{1}\right)_{i}^{n}+\frac{\Delta t}{2 \Delta x}\left\{\left|\left(u_{I}\right)_{i+1 / 2}^{n}\right|\left(\left(\alpha_{1}\right)_{i+1}^{n}-\left(\alpha_{1}\right)_{i}^{n}\right)\right. \\
\left.-\left|\left(u_{I}\right)_{i-1 / 2}^{n}\right|\left(\left(\alpha_{1}\right)_{i}^{n}-\left(\alpha_{1}\right)_{i-1}^{n}\right)\right\}- \\
-\frac{\Delta t}{\Delta x}\left\{\left(u_{I}\right)_{i-1 / 2}^{n}\left(\left(\alpha_{1}\right)_{i}^{n}-\left(\alpha_{1}\right)_{i-1}^{n}\right)+\left(u_{I}\right)_{i+1 / 2}^{n}\left(\left(\alpha_{1}\right)_{i+1}^{n}-\left(\alpha_{1}\right)_{i}^{n}\right)\right\}
\end{array}\right.
$$

or

$$
\left(\alpha_{1}\right)_{i}^{n+1 / 2}=\left(\alpha_{1}\right)_{i}^{n}-\frac{\Delta t}{\Delta x}\left\{\left(u_{I}\right)_{i-1 / 2}^{n,+}\left(\Delta \alpha_{1}\right)_{i-1 / 2}^{n}+\left(u_{I}\right)_{i+1 / 2}^{n,-}\left(\Delta \alpha_{1}\right)_{i+1 / 2}^{n}\right\}
$$

where we have set for all pair $\left(\varphi_{i}^{n}, \varphi_{i+1}^{n}\right)$

$$
(\Delta \varphi)_{i+1 / 2}^{n}=\varphi_{i+1}^{n}-\varphi_{i}^{n}
$$

and used the notations

$$
\varphi^{+}=\max (\varphi, 0), \quad \varphi^{-}=\min (\varphi, 0) .
$$

We thus obtain a natural upwind discretization of the first equation (25). 
Let us next derive the discrete analogues of the phasic mass, momentum and energy conservation equations. We set

$$
\begin{gathered}
\left\{\begin{array}{c}
m_{k}\left(\mathbf{U}_{L}, \mathbf{U}_{R}\right)=\frac{1}{2}\left\{\left(\alpha_{k} \varrho_{k} u_{k}\right)_{L}+\left(\alpha_{k} \varrho_{k} u_{k}\right)_{R}-\right. \\
-\left|\sigma_{k, 1}\right| \alpha_{k, L}\left(\varrho_{k, L}^{*}-\varrho_{k, L}\right)-\left|u_{I}^{*}\right|\left(\alpha_{k, R} \varrho_{k, R}^{*}-\alpha_{k, L} \varrho_{k, L}^{*}\right)- \\
\left.-\left|\sigma_{k, 3}\right| \alpha_{k, R}\left(\varrho_{k, R}-\varrho_{k, R}^{*}\right)\right\},
\end{array}\right. \\
\left\{\begin{array}{c}
q_{k}\left(\mathbf{U}_{L}, \mathbf{U}_{R}\right)=\frac{1}{2}\left\{\left(\alpha_{k} \varrho_{k} u_{k}^{2}\right)_{L}+\left(\alpha_{k} \varrho_{k} u_{k}^{2}\right)_{R}-\right. \\
-\left|\sigma_{k, 1}\right| \alpha_{k, L}\left(\varrho_{k, L}^{*} u_{k, L}^{*}-\varrho_{k, L} u_{k, L}\right)- \\
-\left|u_{I}^{*}\right|\left(\alpha_{k, R} \varrho_{k, R}^{*} u_{k, R}^{*}-\alpha_{k, L} \varrho_{k, L}^{*} u_{k, L}^{*}\right)- \\
\left.-\left|\sigma_{k, 3}\right| \alpha_{k, R}\left(\varrho_{k, R} u_{k, R}-\varrho_{k, R}^{*} u_{k, R}^{*}\right)\right\} \\
-\left|\sigma_{k, 1}\right| \alpha_{k, L}\left(\varrho_{k, L}^{*} e_{k, L}^{*}-\varrho_{k, L} e_{k, L}\right)- \\
-\left|u_{I}^{*}\right|\left(\alpha_{k, R} \varrho_{k, R}^{*} u_{k, R}^{*}-\alpha_{k, L} \varrho_{k, L}^{*} u_{k, L}^{*}\right)- \\
\left.-\left|\sigma_{k, 3}\right| \alpha_{k, R}\left(\varrho_{k, R} e_{k, R}-\varrho_{k, R}^{*} e_{k, R}^{*}\right)\right\}
\end{array}\right.
\end{gathered}
$$

and

$$
\left\{\begin{array}{c}
\left(m_{k}\right)_{i+1 / 2}^{n}=m_{k}\left(\mathbf{U}_{i}^{n}, \mathbf{U}_{i+1}^{n}\right),\left(q_{k}\right)_{i+1 / 2}^{n}=q_{k}\left(\mathbf{U}_{i}^{n}, \mathbf{U}_{i+1}^{n}\right), \\
\left(w_{k}\right)_{i+1 / 2}^{n}=w_{k}\left(\mathbf{U}_{i}^{n}, \mathbf{U}_{i+1}^{n}\right), \\
\left(\Delta m_{k}\right)_{i}^{n}=\left(m_{k}\right)_{i+1 / 2}^{n}-\left(m_{k}\right)_{i-1 / 2}^{n},\left(\Delta q_{k}\right)_{i}^{n}=\left(q_{k}\right)_{i+1 / 2}^{n}-\left(q_{k}\right)_{i-1 / 2}^{n}, \\
\left(\Delta w_{k}\right)_{i}^{n}=\left(w_{k}\right)_{i+1 / 2}^{n}-\left(w_{k}\right)_{i-1 / 2}^{n} .
\end{array}\right.
$$

Then the discrete mass conservation equation for the phase $\mathrm{k}$ can be written

$$
\left(\alpha_{k} \varrho_{k}\right)_{i}^{n+1 / 2}=\left(\alpha_{k} \varrho_{k}\right)_{i}^{n}-\frac{\Delta t}{\Delta x}\left(\Delta m_{k}\right)_{i}^{n} .
$$

Next, setting

$$
\left\{\begin{array}{l}
\left.\left.\left(\Delta\left(\alpha_{k} p_{k}\right)\right)_{i}^{n}=\frac{1}{2}\left\{\left(\alpha_{k} p_{k}\right)\right)_{i+1}^{n}-\left(\alpha_{k} p_{k}\right)\right)_{i}^{n}\right\} \\
\left(p_{I} \Delta \alpha_{k}\right)_{i+1 / 2}^{n}=\left(p_{I}\right)_{i+1 / 2}^{n}\left(\Delta \alpha_{k}\right)_{i+1 / 2}^{n} \\
\left(\alpha_{k} \varrho_{k}\right)_{i+1 / 2}^{n}=\frac{1}{2}\left\{\left(\alpha_{k} \varrho_{k}\right)_{i}^{n}+\left(\alpha_{k} \varrho_{k}\right)_{i+1}^{n}\right\}
\end{array}\right.
$$


one can check that the discrete momentum conservation equation reads

$$
\left\{\begin{array}{l}
\left(\alpha_{k} \varrho_{k} u_{k}\right)_{i}^{n+1 / 2}=\left(\alpha_{k} \varrho_{k} u_{k}\right)_{i}^{n}-\frac{\Delta t}{\Delta x}\left\{\left(\Delta q_{k}\right)_{i}^{n}+\left(\Delta\left(\alpha_{k} p_{k}\right)\right)_{i}^{n}\right\}+ \\
+\frac{\Delta t}{2 \Delta x}\left\{\left(p_{I} \Delta \alpha_{k}\right)_{i-1 / 2}^{n}+\left(p_{I} \Delta \alpha_{k}\right)_{i+1 / 2}^{n}\right\}+\frac{\Delta t}{2}\left\{\left(\alpha_{k} \varrho_{k}\right)_{i-1 / 2}^{n} g+\right. \\
\left.+\left(\alpha_{k} \varrho_{k}\right)_{i+1 / 2}^{n} g-\left(K\left(u_{k}-u_{l}\right)\right)_{i-1 / 2}^{n}-\left(K\left(u_{k}-u_{l}\right)\right)_{i+1 / 2}^{n}\right\} .
\end{array}\right.
$$

Similarly, setting in addition

$$
\left\{\begin{array}{l}
\left.\left.\left(\Delta\left(\alpha_{k} p_{k} u_{k}\right)\right)_{i}^{n}=\frac{1}{2}\left\{\left(\alpha_{k} p_{k} u_{k}\right)\right)_{i+1}^{n}-\left(\alpha_{k} p_{k} u_{k}\right)\right)_{i-1}^{n}\right\} \\
\left(p_{I} u_{I} \Delta \alpha_{k}\right)_{i+1 / 2}^{n}=\left(p_{I}\right)_{i+1 / 2}^{n}\left(u_{I}\right)_{i+1 / 2}^{n}\left(\Delta \alpha_{k}\right)_{i+1 / 2}^{n} \\
\left(u_{I} K\left(u_{k}-u_{l}\right)\right)_{i+1 / 2}^{n}=\left(u_{I}\right)_{i+1 / 2}^{n}\left(K\left(u_{k}-u_{l}\right)\right)_{i+1 / 2}^{n}
\end{array}\right.
$$

the discrete energy conservation equation takes the form

$$
\left\{\begin{array}{c}
\left(\alpha_{k} \varrho_{k} e_{k}\right)_{i}^{n+1 / 2}=\left(\alpha_{k} \varrho_{k} e_{k}\right)_{i}^{n}-\frac{\Delta t}{\Delta x}\left\{\left(\Delta w_{k}\right)_{i}^{n}+\left(\Delta\left(\alpha_{k} p_{k} u_{k}\right)\right)_{i}^{n}\right\}+ \\
+\frac{\Delta t}{2 \Delta x}\left\{\left(p_{I} u_{I} \Delta \alpha_{k}\right)_{i-1 / 2}^{n}+\left(p_{I} u_{I} \Delta \alpha_{k}\right)_{i+1 / 2}^{n}\right\}+ \\
+\frac{\Delta t}{2}\left\{\left(\alpha_{k} \varrho_{k} u_{k}\right)_{i-1 / 2}^{n} g+\left(\alpha_{k} \varrho_{k} u_{k}\right)_{i+1 / 2}^{n} g-\right. \\
\left.-\left(u_{I} K\left(u_{k}-u_{l}\right)\right)_{i-1 / 2}^{n}-\left(u_{I} K\left(u_{k}-u_{l}\right)\right)_{i+1 / 2}^{n}\right\}
\end{array}\right.
$$

\subsection{The second step: $\mathbf{U}^{n+1 / 2} \rightarrow \mathbf{U}^{n+1}$}

In order to define the solution $\mathbf{U}^{n+1}=\left(\mathbf{U}_{i}^{n+1}\right)_{i}$ at time $t^{n+1}$, we propose to simply calculate an approximate value at time $\Delta t$ of the vector $\mathbf{U}$ obtained from (32). To do so, each integral is approximated using a rectangle formula, leading to

$$
\left\{\begin{array}{l}
\left(\frac{\alpha_{1}}{1-\alpha_{1}}\right)^{n+1}=\left(\frac{\alpha_{1}}{1-\alpha_{1}}\right)^{n+1 / 2} \exp \left(\frac{\Delta t}{\tau_{p}}\left(\frac{p_{1}-p_{2}}{p_{1}+p_{2}}\right)^{n+1 / 2}\right), \\
\left(\alpha_{k} \varrho_{k}\right)^{n+1}=\left(\alpha_{k} \varrho_{k}\right)^{n+1 / 2}, \\
\left(\alpha_{k} \varrho_{k} u_{k}\right)^{n+1}=\left(\alpha_{k} \varrho_{k} u_{k}\right)^{n+1 / 2}, \\
\left(p_{1}-p_{2}\right)^{n+1}=\left(p_{1}-p_{2}\right)^{n+1 / 2} \exp \left(-\Delta t \Theta^{n+1 / 2}\left(A_{1}-A_{2}\right)^{n+1 / 2}\right), \\
\left(p_{1} p_{2}\right)^{n+1}=\left(p_{1} p_{2}\right)^{n+1 / 2} \exp \left(-\Delta t\left(\sum_{k=1}^{2} \frac{\Theta A_{k}}{p_{k}}\left(p_{k}-p_{l}\right)\right)^{n+1 / 2}\right) .
\end{array}\right.
$$

Again, we refer the reader to [21] for more details. 


\section{Properties of the scheme and open problems}

Our objective in this section is first to check that if we choose $C_{k, L}$ and $C_{k, R}$ large enough, we ensure on the one hand that the waves of the approximate Riemann solver are properly ordered, and on the other hand that the intermediate densities $\varrho_{k, L}^{*}, \varrho_{k, R}^{*}$ and therefore the densities $\varrho_{i}^{n}$ are positive. For the sake of simplicity, we will assume here

$$
C_{k, L}=C_{k, R}=C, \quad k=1,2,
$$

but the analysis can be easily extended to the general case. Note that in practice, we took for all cell $i$

$$
\left(C_{k}\right)_{i}=(1+\epsilon) \max \left(\left(\rho_{k} c_{k}\right)_{i-1},\left(\rho_{k} c_{k}\right)_{i},\left(\rho_{k} c_{k}\right)_{i+1}\right), \quad k=1,2
$$

with $\epsilon$ varying between 0.01 and 0.1 depending on the numerical test cases. We then perform an asymptotic analysis as $C$ tends to $+\infty$. We begin by studying the behavior of $j_{k}, k=1,2$. As a consequence of (68) and (71), we find that $j_{1}$ is solution of

$$
\left\{\begin{array}{l}
\left\{\chi\left(\alpha_{1}\right)_{a}\left(\sigma_{2,3}-\sigma_{2,1}\right)+(1-\chi)\left(\alpha_{2}\right)_{a}\left(\sigma_{1,3}-\sigma_{1,1}\right)\right\} j_{1}= \\
=(1-\chi)\left\{2 C\left(\left(\alpha_{2}\right)_{a}\left(\alpha_{1} u_{1}\right)_{a}-\left(\alpha_{1}\right)_{a}\left(\alpha_{2} u_{2}\right)_{a}\right)+\right. \\
+\left(\alpha_{1}\right)_{a}\left(\Delta\left(\alpha_{2} p_{2}\right)-\tilde{p}_{I} \Delta \alpha_{2}\right)-\left(\alpha_{2}\right)_{a}\left(\Delta\left(\alpha_{1} p_{1}\right)-\tilde{p}_{I} \Delta \alpha_{1}\right)+ \\
+\Delta x\left(\left(K\left(u_{2}-u_{1}\right)_{a}+\left(\left(\alpha_{2}\right)_{a}\left(\alpha_{1} \varrho_{1}\right)_{a}-\left(\alpha_{1}\right)_{a}\left(\alpha_{2} \varrho_{2}\right)_{a}\right) g\right)\right\} .
\end{array}\right.
$$

Since by (51)

$$
\sigma_{k, 3}-\sigma_{k, 1}=\Delta u_{k}+C\left(\frac{1}{\varrho_{k, L}}+\frac{1}{\varrho_{k, R}}\right)=C\left\{2\left(\frac{1}{\varrho_{k}}\right)_{a}+O\left(\frac{1}{C}\right)\right\}
$$

we obtain

$$
\begin{gathered}
2\left\{\chi\left(\alpha_{1}\right)_{a}\left(\frac{1}{\varrho_{2}}\right)_{a}+(1-\chi)\left(\alpha_{2}\right)_{a}\left(\frac{1}{\varrho_{1}}\right)_{a}+O\left(\frac{1}{C}\right)\right\} j_{1}= \\
=(1-\chi)\left(\left(\alpha_{2}\right)_{a}\left(\alpha_{1} u_{1}\right)_{a}-\left(\alpha_{1}\right)_{a}\left(\alpha_{2} u_{2}\right)_{a}\right)+O\left(\frac{1}{C}\right)
\end{gathered}
$$

and therefore

$$
j_{1}=O(1), \quad j_{2}=O(1)
$$

Next, using (70), we get

$$
u_{i}^{*}=\left(\frac{1}{\varrho_{1}}\right)_{a} j_{1}+\left(\frac{1}{\varrho_{2}}\right)_{a} j_{2}+\left(\alpha_{1} u_{1}\right)_{a}+\left(\alpha_{2} u_{2}\right)_{a}+O\left(\frac{1}{C}\right)=O(1) .
$$

Hence we have for $C$ large enough

$$
\sigma_{k, 1}=-\frac{C}{\varrho_{k, L}}+O(1)<u_{I}^{*}=O(1)<\sigma_{k, 3}=\frac{C}{\varrho_{k, R}}+O(1), \quad k=1,2
$$


so that the waves are indeed well ordered. On the other hand, (60) gives

$$
\frac{1}{\varrho_{k, L}^{*}}=\frac{1}{\varrho_{k, L}}+\frac{1}{C}\left(u_{k, L}^{*}-u_{k, L}\right), \quad \frac{1}{\varrho_{k, R}^{*}}=\frac{1}{\varrho_{k, R}}-\frac{1}{C}\left(u_{k, R}^{*}-u_{k, R}\right) .
$$

Together with (73), this yields

$$
\begin{aligned}
& \frac{1}{\varrho_{k, L}^{*}}=\frac{1}{\varrho_{k, L}}\left(1+\frac{j_{k}+\alpha_{k, L} \varrho_{k, L}\left(u_{I}^{*}-u_{k, L}\right)}{C \alpha_{k, L}-j_{k}}\right)=\frac{1}{\varrho_{k, L}}\left(1+O\left(\frac{1}{C}\right)\right), \\
& \frac{1}{\varrho_{k, R}^{*}}=\frac{1}{\varrho_{k, R}}\left(1-\frac{j_{k}+\alpha_{k, R} \varrho_{k, R}\left(u_{I}^{*}-u_{k, R}\right)}{C \alpha_{k, R}+j_{k}}\right)=\frac{1}{\varrho_{k, R}}\left(1+O\left(\frac{1}{C}\right)\right)
\end{aligned}
$$

which proves the positivity of $\varrho_{k, L}^{*}$ and $\varrho_{k, R}^{*}$ again for $C$ large enough.

We can also state obvious properties satisfied by the numerical scheme. First, the void fractions $\left(\alpha_{k}\right)_{i}^{n}$ necessarily remain in the expected interval $[0,1]$ since they are simply transported by the approximate Riemann solver. Then, the stationary coupling waves between the two phases, that is the ones associated with velocity $u_{I}$, are natural solutions of the approximate Riemann solver when the sources are neglected. Thus, they are exactly captured by the numerical scheme. Of course, non stationary coupling waves are also natural solutions of the approximate Riemann solver but as usual are not exactly captured by the scheme due to the averaging procedures inherent to the method. At last, let us mention than the numerical scheme is by construction conservative on the partial mass $\alpha_{k} \rho_{k}, k=1,2$, on the total momentum $\alpha_{1} \rho_{1} u_{1}+\alpha_{2} \rho_{2} u_{2}$ and on the total energy $\alpha_{1} \rho_{1} E_{1}+\alpha_{2} \rho_{2} E_{2}$.

It would be also interesting to prove the positivity of the specific internal energies $\left(\varepsilon_{k}\right)_{i}^{n}$, and the validity of a discrete entropy inequality related to (17). These issues remain open problems at the moment. Let us mention however that similarly to the continuous level, we suspect that a relevant definition of $\tilde{p}_{I}$ (here given by (78)-(79)) could yield the expected entropy inequality.

At last, the asymptotic-preserving property of the scheme which has motivated the whole numerical strategy and in particular the upwind treatment of the sources including them in the definition of the approximate Riemann solver (see again [10] for a very similar approach which turned out to be successfull in a simplified context), has not been proved theoretically. However, we will observe in the next section devoted to the numerical experiments that we can actually recover a pretty good approximation of the asymptotic drift velocity (24).

\section{$7 \quad$ Numerical results}

We propose in this section various test cases in order to assess the proposed algorithm. We begin by considering Riemann problems, for which exact solutions are available, and compare the numerical results with the ones provided by alternate methods available in the literature. More precisely, we will consider the HLL-type method proposed by Saurel \& Abgrall [37], the strategy presented by Andrianov, Saurel \& Warnacke [4], the VFRoe scheme derived by Gallouët, 
Hérard \& Seguin [21], and at last the Godunov scheme of Schwendeman, Wahle \& Kapila [40]. Note from now on that the comparison will be based on simulations and pictures proposed in these papers only.

We will then go on considering more typical two-phase flow simulations, namely the Ransom water faucet problem, the sedimentation test case, a desequilibrium in velocities test case, and at last a vertical bubbly column. These simulations involve non zero source terms which allows to assess our numerical upwind strategy for the external and drag forces. In particular, in the last test case, a specific attention will be paid to the (numerical) asymptotic preserving property of the scheme.

Except otherwise stated, all our simulations are performed on the interval $[0,1]$ and the (possible) Riemann initial discontinuity is always located at point $x=0.5$. Our numerical scheme will be referred to as Godunov-type scheme in the following pictures.

\subsection{Riemann problems}

We consider in this part a Riemann initial data

$$
\mathbf{U}(x, 0)=\mathbf{U}_{0}(x)=\left\{\begin{array}{lll}
\mathbf{U}_{L} & \text { if } & x<0, \\
\mathbf{U}_{R} & \text { if } & x>0,
\end{array}\right.
$$

where for the sake of clarity, the initial states $\mathbf{U}_{L}$ and $\mathbf{U}_{R}$ will be defined from the following set of initial values in primitive variables : $\alpha_{1}, \rho_{k}, u_{k}$ and $p_{k}$, $k=1,2$. Regarding the closure laws, we take just for simplicity the classical ideal gas equation of state

$$
p_{k}=\left(\gamma_{k}-1\right) \rho_{k} \varepsilon_{k}, k=1,2,
$$

with $\gamma=\gamma_{1}=\gamma_{2}=1.4$.

\subsubsection{Test 1 : isolated coupling wave}

In the first two simulations, we consider the simple situation of an isolated coupling wave propagating with velocity $u_{I}$. The first simulation (Test 1a) is taken from [21] and corresponds to the choice $\chi=0.5$, that is

$$
u_{I}=\frac{\alpha_{1} \rho_{1} u_{1}+\alpha_{2} \rho_{2} u_{2}}{\alpha_{1} \rho_{1}+\alpha_{2} \rho_{2}} .
$$

We choose

$$
\begin{array}{lll}
\alpha_{1, L}=0.9, & \left(\rho_{1}, u_{1}, p_{1}\right)_{L}=\left(1,100,10^{5}\right) & \left(\rho_{2}, u_{2}, p_{2}\right)_{L}=\left(1,100,10^{5}\right) \\
\alpha_{1, R}=0.5, & \left(\rho_{1}, u_{1}, p_{1}\right)_{R}=\left(0.125,100,10^{5}\right) & \left(\rho_{2}, u_{2}, p_{2}\right)_{R}=\left(0.125,100,10^{5}\right) .
\end{array}
$$

Solutions are presented on Fig. 1 and the results are compared with the ones given by the VFRoe scheme [21]. We note that both schemes preserve the constant velocities and pressures by construction, and present exactly the same numerical diffusion. Fig. 2 shows the solutions provided by our scheme on several meshes.

The second simulation (Test 1b) still corresponds to an isolated coupling wave, but now associated with the choice $\chi=1$, leading to $u_{I}=u_{1}$. Initial conditions 

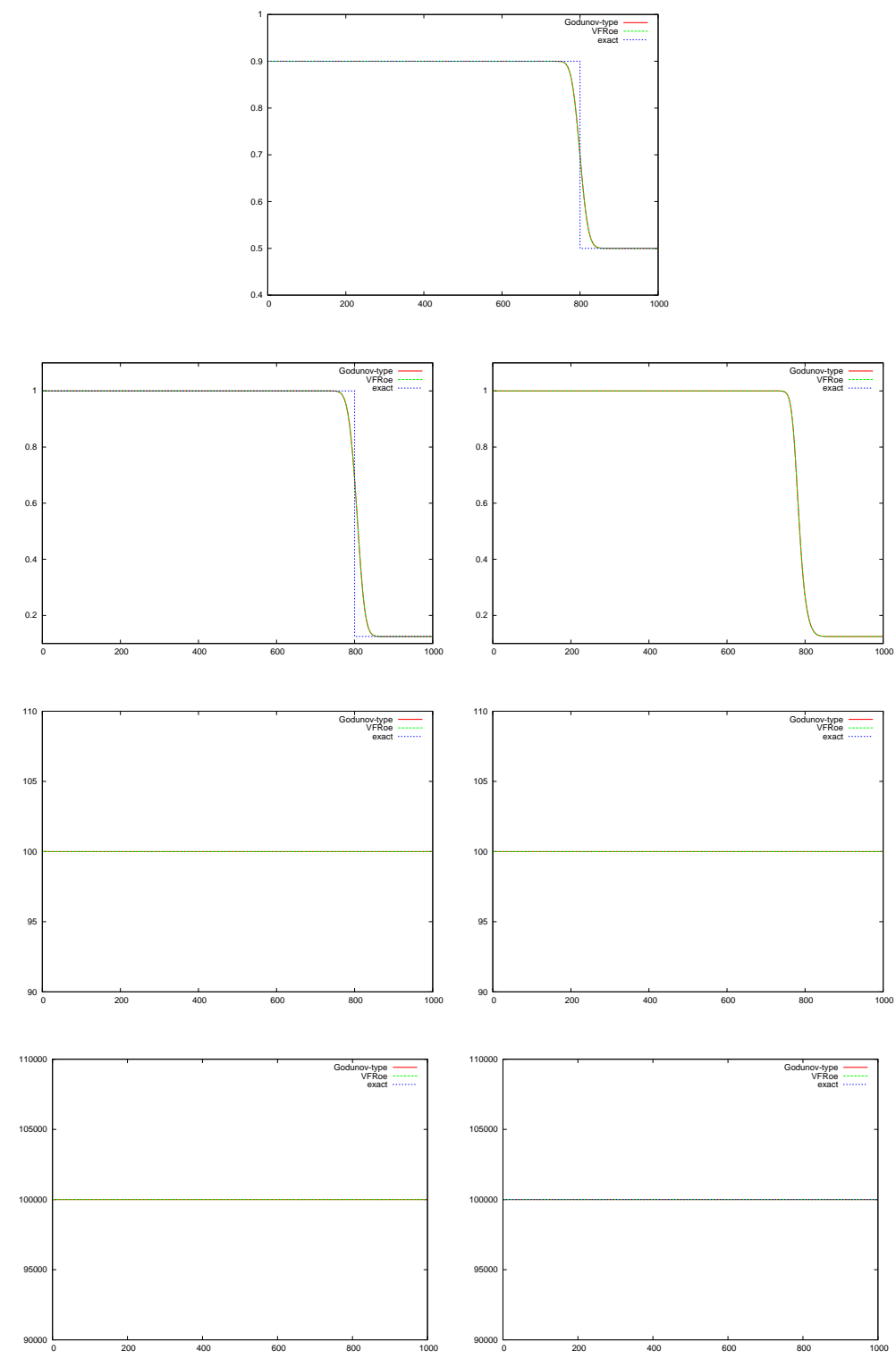

Figure 1: Comparison between exact and numerical solutions of Test 1a at time $t=3$ and for a 1000-point mesh. From the top to the bottom right : $\mathrm{x}$ versus $\alpha_{1}, \rho_{1}, \rho_{2}, u_{1}, u_{2}, p_{1}, p_{2}$ 

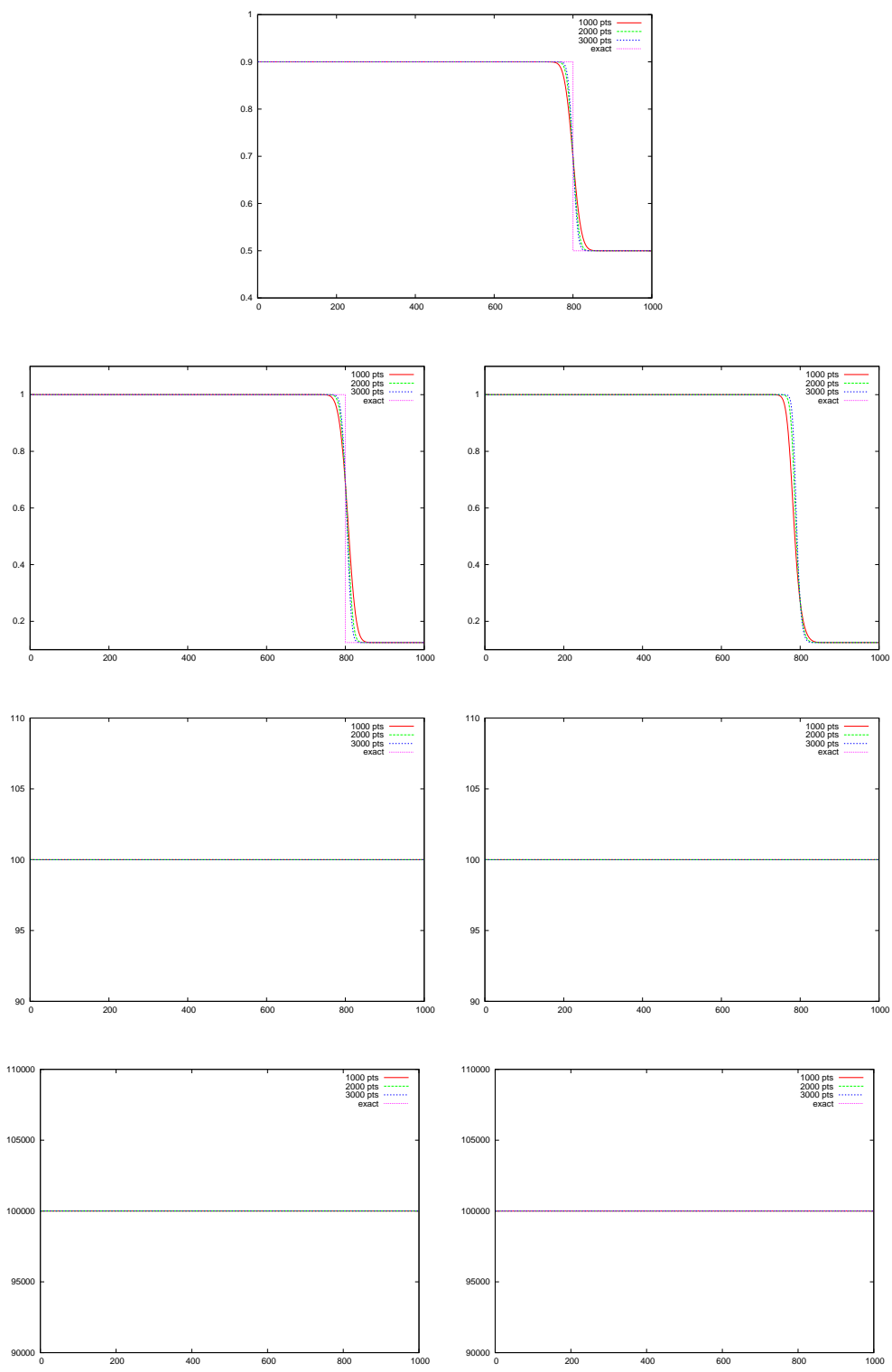

Figure 2: Comparison between exact and numerical solutions of Test 1a at time $t=3$ and for several mesh sizes. From the top to the bottom right : $\mathrm{x}$ versus $\alpha_{1}, \rho_{1}, \rho_{2}, u_{1}, u_{2}, p_{1}, p_{2}$ 
are taken from [5] and given by

$$
\begin{array}{lll}
\alpha_{1, L}=0.8, & \left(\rho_{1}, u_{1}, p_{1}\right)_{L}=(2,0.3,5) & \left(\rho_{2}, u_{2}, p_{2}\right)_{L}=(1,2,1) \\
\alpha_{1, R}=0.3, & \left(\rho_{1}, u_{1}, p_{1}\right)_{R}=(2,0.3,12.8567) & \left(\rho_{2}, u_{2}, p_{2}\right)_{R}=(0.1941,2.8011,0.1) .
\end{array}
$$

We note that the pressures and velocity $u_{2}$ are not taken to be equal anymore. Solutions are presented on Fig. 3. We observe that the results are in good agreement with the exact solutions. They also compare very well with the ones given in [5].The right state of the coupling wave, in particular for the $u_{2}$ component, agrees well with the exact value. The small amplitude oscillations observed on $\rho_{1}$ and $u_{1}$ are classical and due to the initial pressures and velocity $u_{2}$ desequilibrium. Fig. 4 shows the numerical solutions obtained with several mesh sizes.

\subsubsection{Test 2 : a general Riemann problem}

As a general Riemann problem, we consider here a test case taken from [40] for which the exact solution is known (see Table 1, page 499) and comparisons with other methods from the literature are available.

Initial conditions are such that the mixture is at rest and presents a (negative) jump in the volume fraction $\alpha_{1}$. The density $\rho_{1}$ and pressure $p_{1}$ are equal on each side of the initial discontinuity. More precisely, the initial states $\mathbf{U}_{L, R}$ are calculated from the following values in primitive variables :

$$
\begin{aligned}
& \alpha_{1, L}=0.8, \quad\left(\rho_{1}, u_{1}, p_{1}\right)_{L}=(1,0,1) \quad\left(\rho_{2}, u_{2}, p_{2}\right)_{L}=(0.2,0,0.3) \\
& \alpha_{1, R}=0.3, \quad\left(\rho_{1}, u_{1}, p_{1}\right)_{R}=(1,0,1) \quad\left(\rho_{2}, u_{2}, p_{2}\right)_{L}=(1,0,1) .
\end{aligned}
$$

leading to an exact solution made of a combination of shock, contact discontinuity and rarefaction waves, in addition to the expected coupling wave associated with $u_{I}=u_{1}$. All the solutions are plotted at time $t=0.2$.

We first give on Fig. 5 the results obtained by our algorithm with a mesh composed of 200, 400 and 800 cells and compare the numerical and exact solutions. We observe that the phase 2 presents a good agreement while the phase 1 suffers from overshoots and undershoots at the extremes waves. However, we note that the void fraction, and more generally the coupling wave, is very well computed in the sense that the left and right states associated with $u_{I}$-discontinuity agree with the exact values. In order to illustrate this good property, we proceed as in [40] and compare on Fig. 6 the behavior of the numerical solutions close to the coupling wave with that determined by the exact jump conditions across this wave. These are provided by the five $u_{I}$-Riemann invariants, given in this 

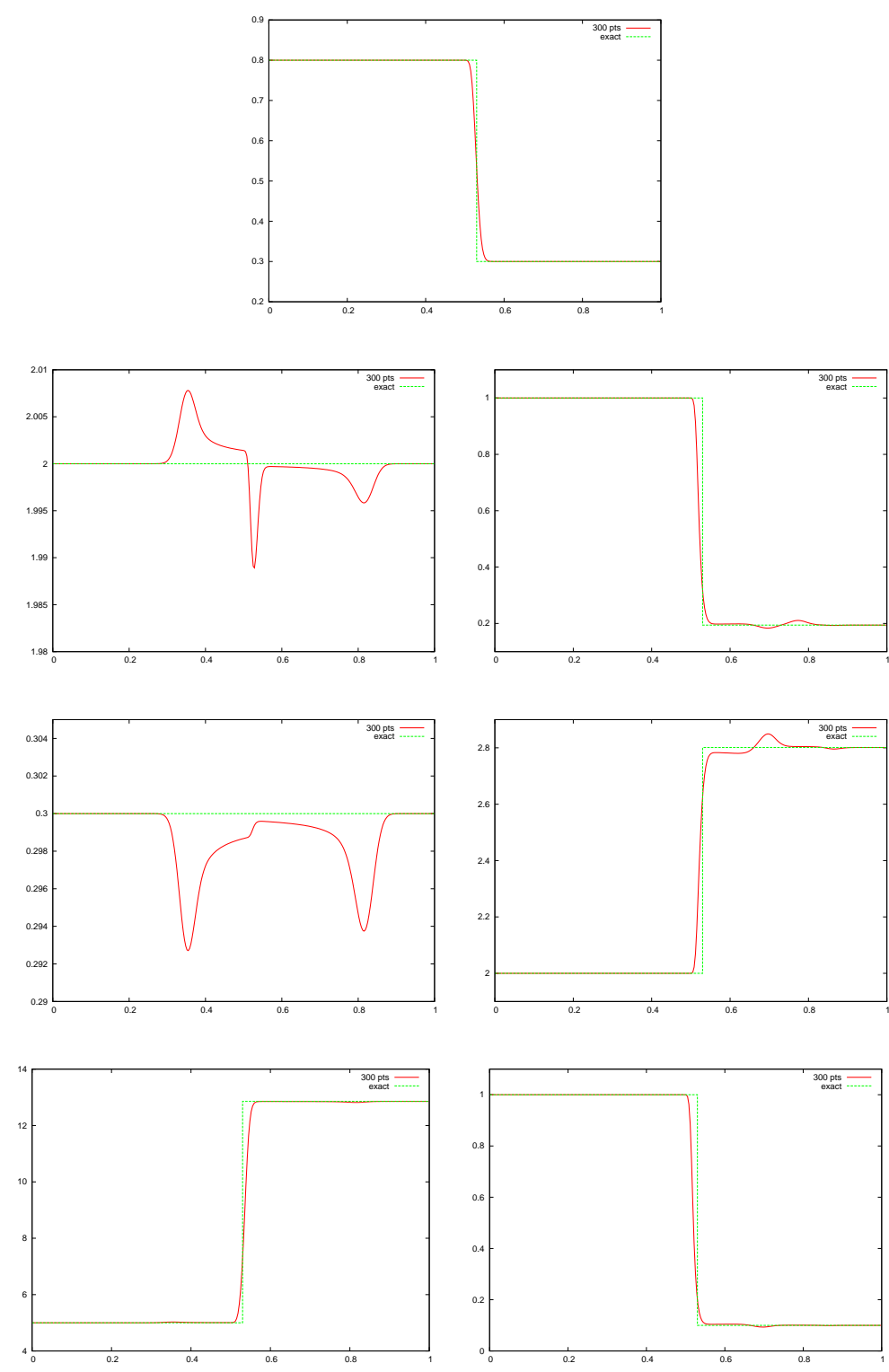

Figure 3: Comparison between exact and numerical solutions of Test $1 \mathrm{~b}$ at time $t=0.1$ and for a 300-point mesh. From the top to the bottom right : $\mathrm{x}$ versus $\alpha_{1}, \rho_{1}, \rho_{2}, u_{1}, u_{2}, p_{1}, p_{2}$ 

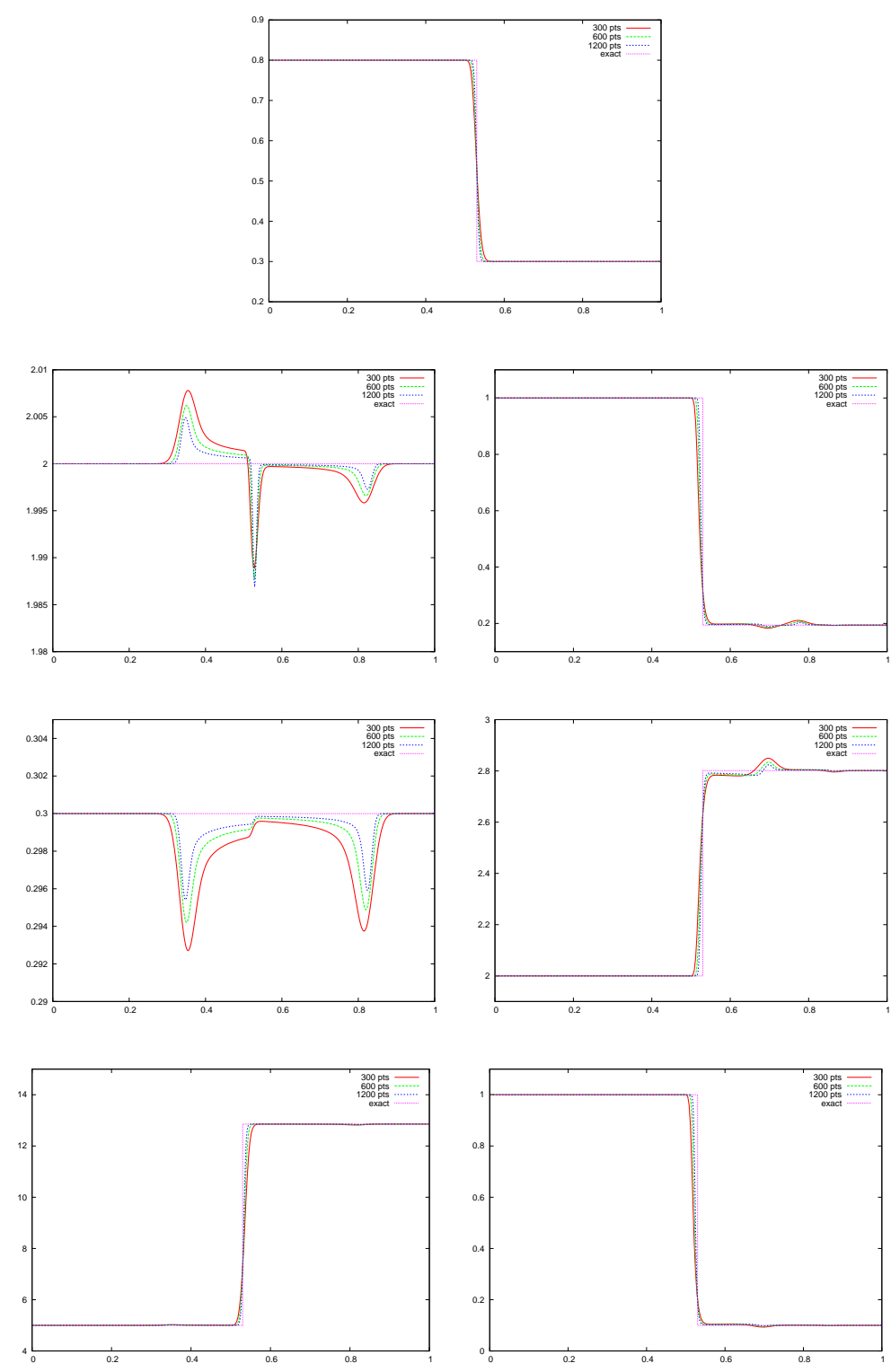

Figure 4: Comparison between exact and numerical solutions of Test $1 \mathrm{~b}$ at time $t=0.1$ and for several mesh sizes. From the top to the bottom right : $\mathrm{x}$ versus $\alpha_{1}, \rho_{1}, \rho_{2}, u_{1}, u_{2}, p_{1}, p_{2}$ 
case by (see again [40]) :

$$
\left\{\begin{array}{l}
K_{0}=u_{I}=u_{1} \\
K_{1}=\left(1-\alpha_{1}\right) \rho_{2}\left(u_{2}-u_{I}\right) \\
K_{2}=\left(1-\alpha_{1}\right) \rho_{2}\left(u_{2}-u_{I}\right)^{2}+\alpha_{1} p_{1}+\left(1-\alpha_{1}\right) p_{2} \\
K_{3}=\frac{\gamma p_{2}}{(\gamma-1) \rho_{2}}+\frac{1}{2}\left(u_{2}-u_{I}\right)^{2} \\
K_{4}=\frac{p_{2}}{\rho_{2}^{\gamma}}
\end{array}\right.
$$

For $\alpha_{1}$ varying from $\alpha_{1, L}$ to $\alpha_{1, R}$, these equations provide a parametrization of the exact (vanishingly thin) layer of the $u_{I}$ coupling wave. This one is compared with the numerical layer induced by the numerical diffusion of the scheme. Note that the five constants $K_{i}, i=0, \ldots, 4$ are calculated using the exact value of the right state corresponding to $\alpha_{1}=\alpha_{1, R}$ as given in [40]. As expected, Fig. 6 shows a good agreement between exact and numerical layers.

We now compare our numerical solutions on Fig. 7 with the ones proposed by Saurel and Abgrall [37] (referred to as $G_{H L L}$ ), by Andrianov et al. [4] (referred to as $G_{A S W}$ ) and by Schwendeman et al. [40] (referred to as $G_{1}$ ) on Fig. 8. Notice that the latter one corresponds to an exact Godunov method (i.e. based on the exact resolution of the Riemann problem) and is thus expected to be the most precise. This is actually the case, we indeed observe that with this method, all the intermediate states are perfectly computed and that the solutions do not present any odd behavior next to the acoustic waves. As far as the other methods are concerned, we first observe that the $G_{H L L}$ method proposed by Saurel and Abgrall [37] is the most diffusive on this test case. The $G_{A S W}$ method of Andrianov et al. [4] is less diffusive but does not provide us with a very good approximation of the constant states of the $u_{I}$ coupling wave. On the contrary, we can observe that our approach behaves very well in the vicinity of this coupling wave (while still presenting overshoots and undershoots next to the extreme waves of the solution). In particular, the left and right states of the $u_{I}$-contact wave appears to be better evaluated.

\subsubsection{Test 3 : a general Riemann problem with several values of $\chi$}

To concude this first batch of test cases, let us emphasize that our algorithm allows by construction to deal with several values of the interfacial velocity $u_{I}$ and pressure $p_{I}$, depending on the underlying modelling assumptions. In particular, we have seen that admissible values for the couple $\left(u_{I}, p_{I}\right)$ may be parametrized by the parameter $\chi \in[0,1]$. We propose now to take advantage of this flexibility of our algorithm to compare the solutions obtained for several values of $\chi$. The Riemann initial data is the same as for Test 2 . So far, we dealt with $\chi=1$ for the sake of comparison with the results presented in [37], [4], [40]. We now also consider the natural values $\chi=0$ and $\chi=0.5$. The results are presented on Fig. 9. We observe in particular that the values $\chi=0$ and $\chi=0.5$ now present an even behavior in the vicinity of the acoustic waves (unlike the value $\chi=1$ ). 

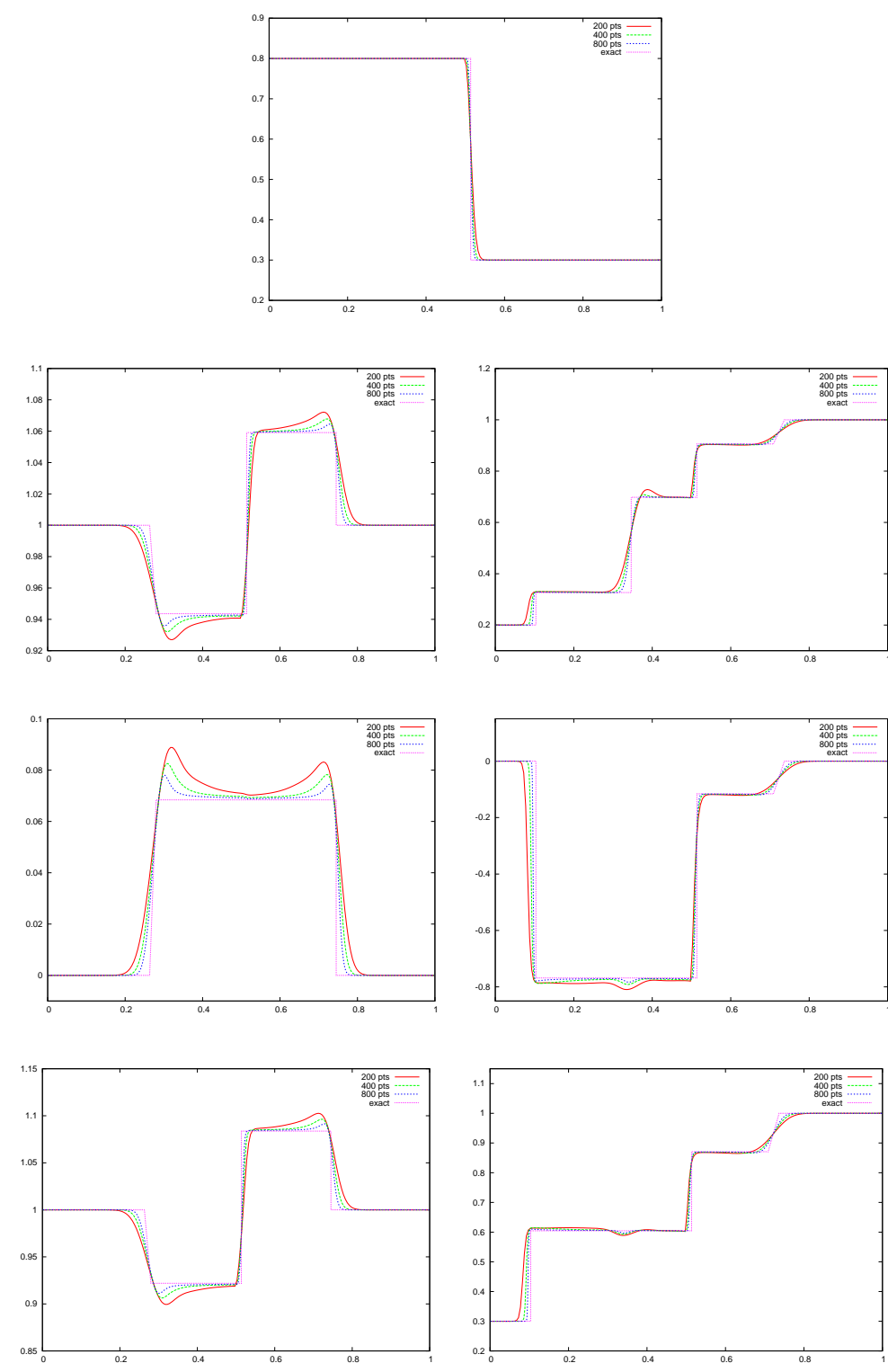

Figure 5: Comparison between exact and numerical solutions of Test 2 at time $t=0.2$ and for several mesh sizes. From the top to the bottom right: $\mathrm{x}$ versus $\alpha_{1}, \rho_{1}, \rho_{2}, u_{1}, u_{2}, p_{1}, p_{2}$ 

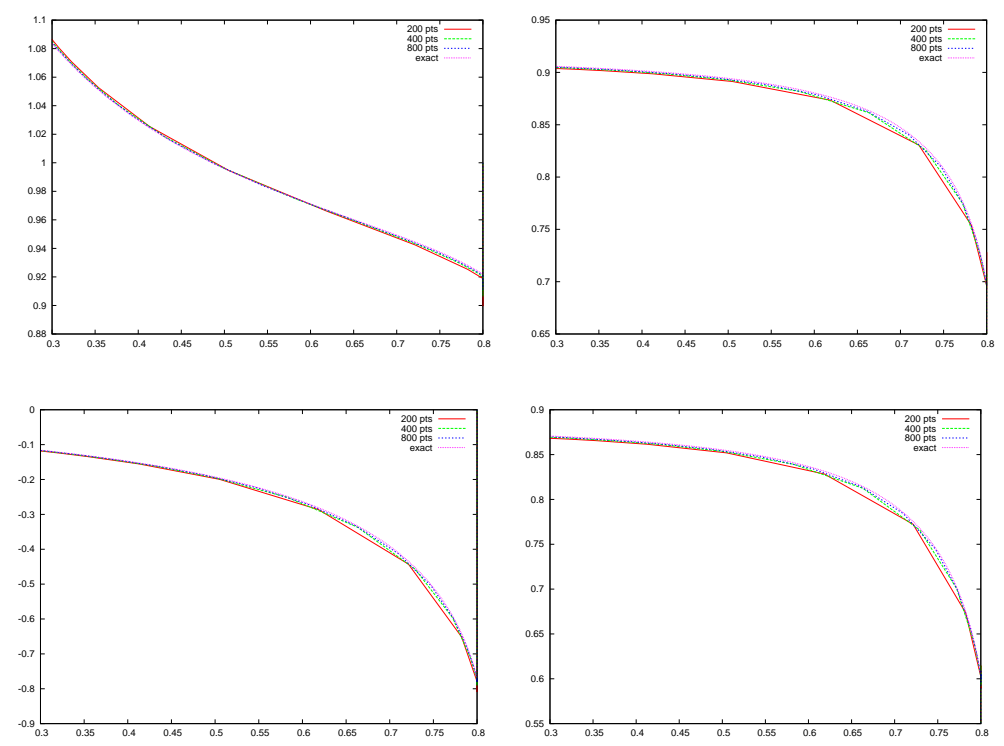

Figure 6: Behaviour of $p_{1}, \rho_{2}, u_{2}, p_{2}$ (from the top left to the bottom right) versus $\alpha_{1}$ through the $u_{I}$ contact layer at time $t=0.2$ and for Test 2
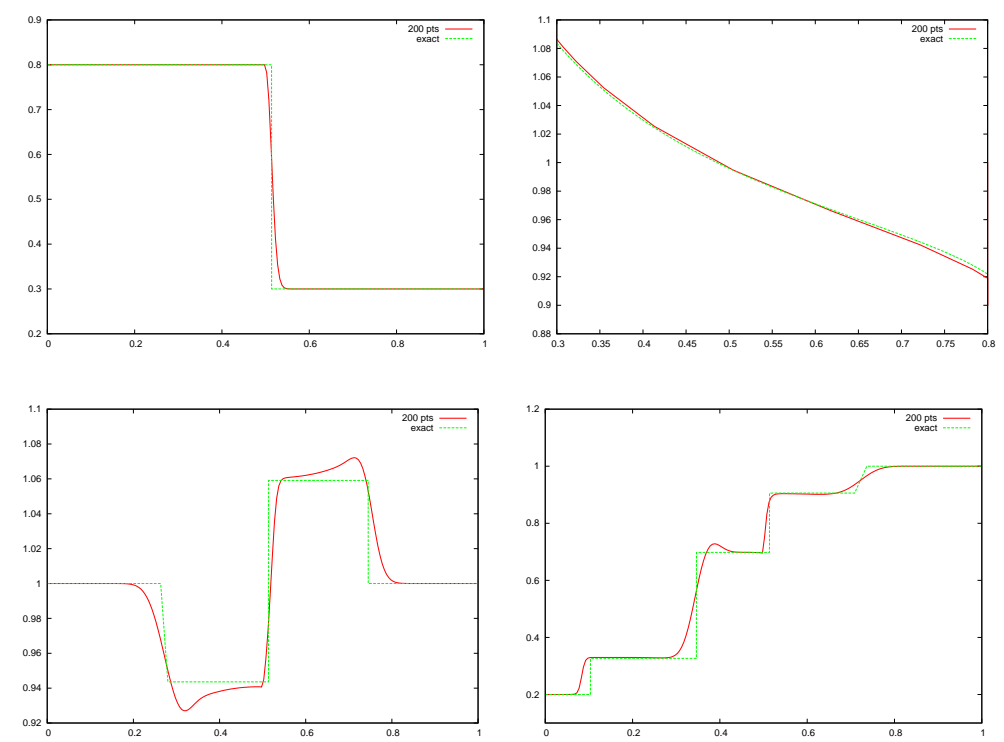

Figure 7: Behaviour of $\alpha_{1}(x), p_{1}\left(\alpha_{1}\right), \rho_{1}(x), \rho_{2}(x)$ at time $t=0.2$ and for Test 2 

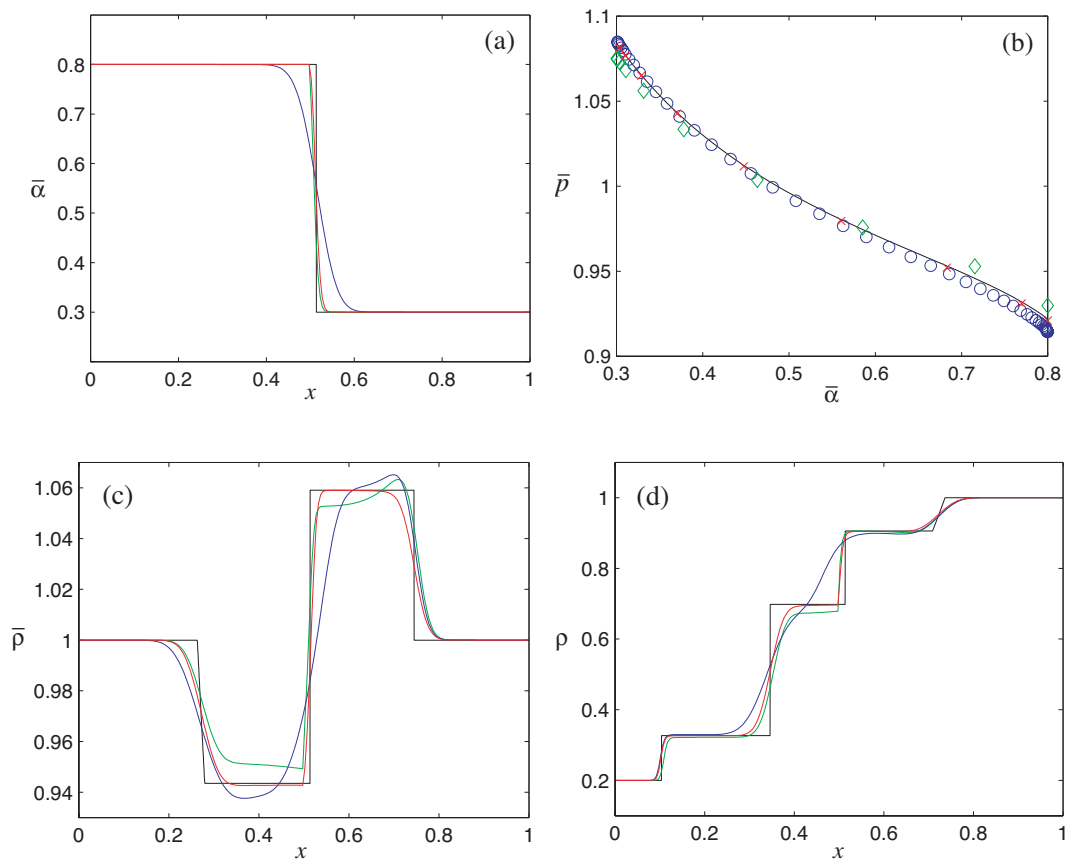

Figure 8: Behaviour of $\alpha_{1}(x), p_{1}\left(\alpha_{1}\right), \rho_{1}(x), \rho_{2}(x)$ at time $t=0.2$ and for Test 2 . These pictures are taken from [40] by courtesy of the authors. The curves are given by method $G_{H L L}$ (blue), $G_{A S W}$ (green) and $G_{1}$ (red). The exact solution appears in black. For interpretation of the references to color in this figure legend, the reader is referred to the web version of this article 
Above all, we note that the three solutions are actually different, highlighting the importance of having a scheme capable of dealing with various values of $\chi$.

Remark: We have not been able to pass the latter test case with the VFRoe scheme proposed in [21], probably due to the lack of positivity properties of the VFRoe approach. On the contrary, let us mention that unlike our approach the VFRoe scheme turns out to be able to compute solutions involving the resonance phenomenon, see Test 2 in [21]. Recall indeed that we have assumed here from the beginning that the waves are ordered such that the acoustic waves propagate faster. More generally, if we consider Riemann initial data such that both our approach and the VFRoe scheme succeed in computing an approximate solution, we observed a good agreement between them. It is worth noticing however that the numerical diffusion turned out to be equivalent for both scheme across the coupling wave (as observed in Test 1a above) and the acoustic waves, but a little less important for the VFRoe scheme across the contact discontinuities associated with $u_{1}$ an $u_{2}$. This is clearly expected since these contact discontinuities are not present in our approximate Riemann solver. These numerical results are not reported here.

\subsection{Influence of the source terms}

Let us now take into account the source terms effects in our simulations. We begin this section with the well-known Ransom faucet and sedimentation problems where only the external forces (namely the gravity) and the relaxation pressure term in the void fraction equation are accounted for. The next simulation considers only the interfacial drag force and the relaxation pressure term. The fourth one involves both the external and drag forces and the relaxation pressure term, and focus on the asymptotic behavior of the model under consideration when the underlying relaxation times are small.

We consider again just for simplicity the classical ideal gas equation of state

$$
p_{k}=\left(\gamma_{k}-1\right) \rho_{k} \varepsilon_{k}, \quad k=1,2,
$$

but now with $\gamma_{1}=1.0005$ and $\gamma_{2}=1.4$.

In order to define the interfacial velocity and pressure, we take $\chi=0.5$.

As far as the relaxation pressure term is concerned, we take

$$
K_{P}(\mathbf{U})=\frac{1}{\tau_{p}} \frac{\alpha_{1}\left(1-\alpha_{1}\right)}{p_{1}+p_{2}}\left(p_{1}-p_{2}\right),
$$

where the relaxation time $\tau_{p}$ has to be specified.

At last, the forthcoming test cases involves boundary conditions at the end points $x_{\min }$ and $x_{\max }$ of the domain $\left(x_{\min }=0\right.$ and $x_{\max }=1$ except otherwise stated). Regarding the numerical treatment of these boundary conditions, we have used two classical fictitious states we call here $\mathbf{U}_{\min }$ and $\mathbf{U}_{\max }$. They will be precised for each test case.

\subsubsection{Test 4 : the Ransom water faucet problem}

This test case is a very classical benchmark test [35]. The configuration corresponds to a $12 \mathrm{~m}$ long vertical tube $\left(x_{\min }=0\right.$ and $\left.x_{\max }=12\right)$. The initial 

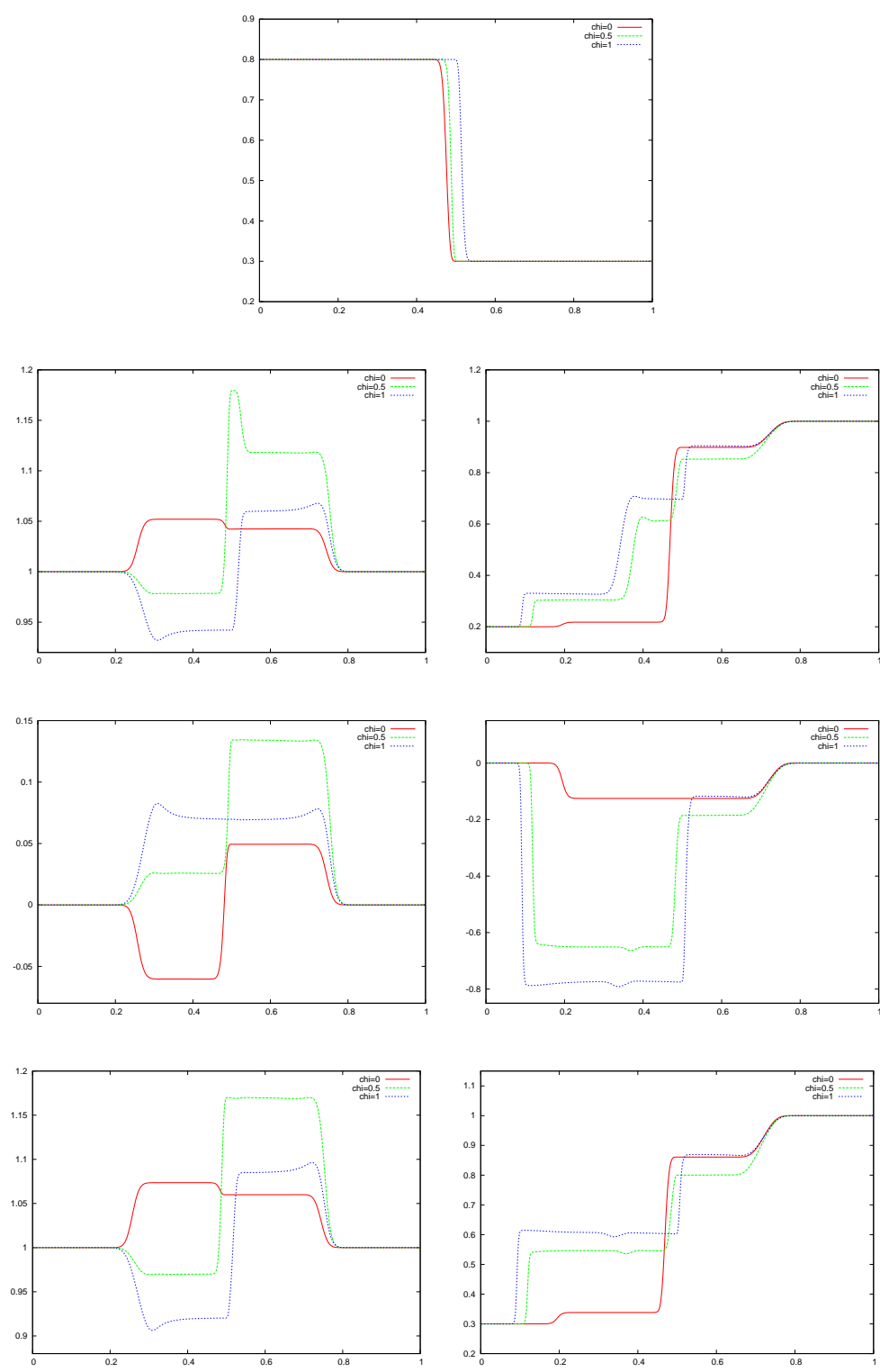

Figure 9: Comparison between numerical solutions of Test 3 at time $t=0.2$ and for several values of $\chi$. The mesh is made of 400 points. From the top to the bottom right : x versus $\alpha_{1}, \rho_{1}, \rho_{2}, u_{1}, u_{2}, p_{1}, p_{2}$ 
condition conists of a uniform column of water (indexed by 1) in the air (indexed by 2). The void fraction is such that $\alpha_{1}=0.8$. The velocities $u_{1}$ and $u_{2}$ are also uniform and respectively equal to $10 \mathrm{~m} / \mathrm{s}$ and $0 \mathrm{~m} / \mathrm{s}$. All the pressures are set to be equal to $10^{5} \mathrm{~Pa}$. Initial densities are such that $\rho_{1}=10^{3} \mathrm{~kg} / \mathrm{m}^{3}$ and $\rho_{2}=1 \mathrm{~kg} / \mathrm{m}^{3}$. Since the constant gravity field $\mathrm{g}=9.81 \mathrm{~m} / \mathrm{s}^{2}$ is taken into account, the flow is driven by the boundary conditions given by $\alpha_{1}(0, t)=0.8$, $u_{1}(0, t)=10 \mathrm{~m} / \mathrm{s}, u_{2}(0, t)=0 \mathrm{~m} / \mathrm{s}, \rho_{1}(0, t)=10^{3} \mathrm{~kg} / \mathrm{m}^{3}, \rho_{2}(0, t)=1 \mathrm{~kg} / \mathrm{m}^{3}$ and $p_{1}(12, t)=p_{2}(12, t)=10^{5} \mathrm{~Pa}$. From a numerical point of view, the fictitious states are such that

$$
\alpha_{1, \min }=0.8, \quad\left(\rho_{1}, u_{1}, p_{1}\right)_{\min }=\left(10^{3}, 10,10^{5}\right), \quad\left(\rho_{2}, u_{2}, p_{2}\right)_{\min }=\left(1,0,10^{5}\right)
$$

and

$$
p_{1, \max }=p_{2, \max }=10^{5} .
$$

Classical Neumann numerical boundary conditions are applied to define

$$
\alpha_{1, \max }, \quad\left(\rho_{1}, u_{1}\right)_{\max }, \quad\left(\rho_{2}, u_{2}\right)_{\max }
$$

(the values are taken from inside the domain). At last, the time scale is $\tau_{p}=510^{-4} s$ in the pressure relaxation term.

The numerical results are plotted on Fig. 10 and compared with the ones given by the VFRoe scheme proposed in [21]. Solutions are very similar. On Fig. 11 are superimposed the results obtained with our scheme for various mesh sizes. We note in particular that an usual problem encountered when dealing with a two-fluid one-pressure model is related to the presence of complex wave speeds when the mesh size becomes too small (approximatively more than 1000 cells, see [21]) and the appearance of negative volume fractions. Here, the two-fluid two-pressure model under consideration is always (possibly weakly) hyperbolic. The finest mesh we have considered here is made of 20000 cells and did not raise any difficulty.

\subsubsection{Test 5 : the sedimentation test case}

Again, this test case is a classical benchmark test for the simulation of twophase flows [12]. The configuration corresponds to a $7.5 \mathrm{~m}$ long vertical tube, that is $x_{\min }=0$ and $x_{\max }=7.5$. The initial condition conists of a uniform mixture of liquid (indexed by 1) and gas (indexed by 2) such that $\alpha_{1}=0.5$. The initial velocities $u_{1}$ and $u_{2}$ are equal to $0 \mathrm{~m} / \mathrm{s}$, and both pressures are equal with $p_{1}=p_{2}=10^{5} \mathrm{~Pa}$. Initial densities are such that $\rho_{1}=10^{3} \mathrm{~kg} / \mathrm{m}^{3}$ and $\rho_{2}=1 \mathrm{~kg} / \mathrm{m}^{3}$. The domain is closed, meaning that classical rigid wall boundary conditions are imposed at the boundaries : $u_{k}\left(x_{\min }, t\right)=u_{k}\left(x_{\max }, t\right)=0$, $k=1,2$. Since the constant gravity field $g=9.81 \mathrm{~m} / \mathrm{s}^{2}$ is present, both phases separate and the solution at time $t=+\infty$ is made of a two regions of pure liquid (at the bottom of the tube, $\alpha_{1}\left(x_{\max },+\infty\right)=1$ ) and gas (at the top of the tube, $\left.\alpha_{1}\left(x_{\min },+\infty\right)=0\right)$. From a numerical point of view and as it is usual, the fictitious states are obtained from classical numerical Neumann boundary conditions applied to the primitive variables, except for the velocities $u_{k, \min }$ and $u_{k, \max }$ that are classically given the opposite values of the velocities $u_{k}$ coming from inside the domain $(k=1,2)$.

At last, let us mention that in order to avoid natural numerical instabilities due 

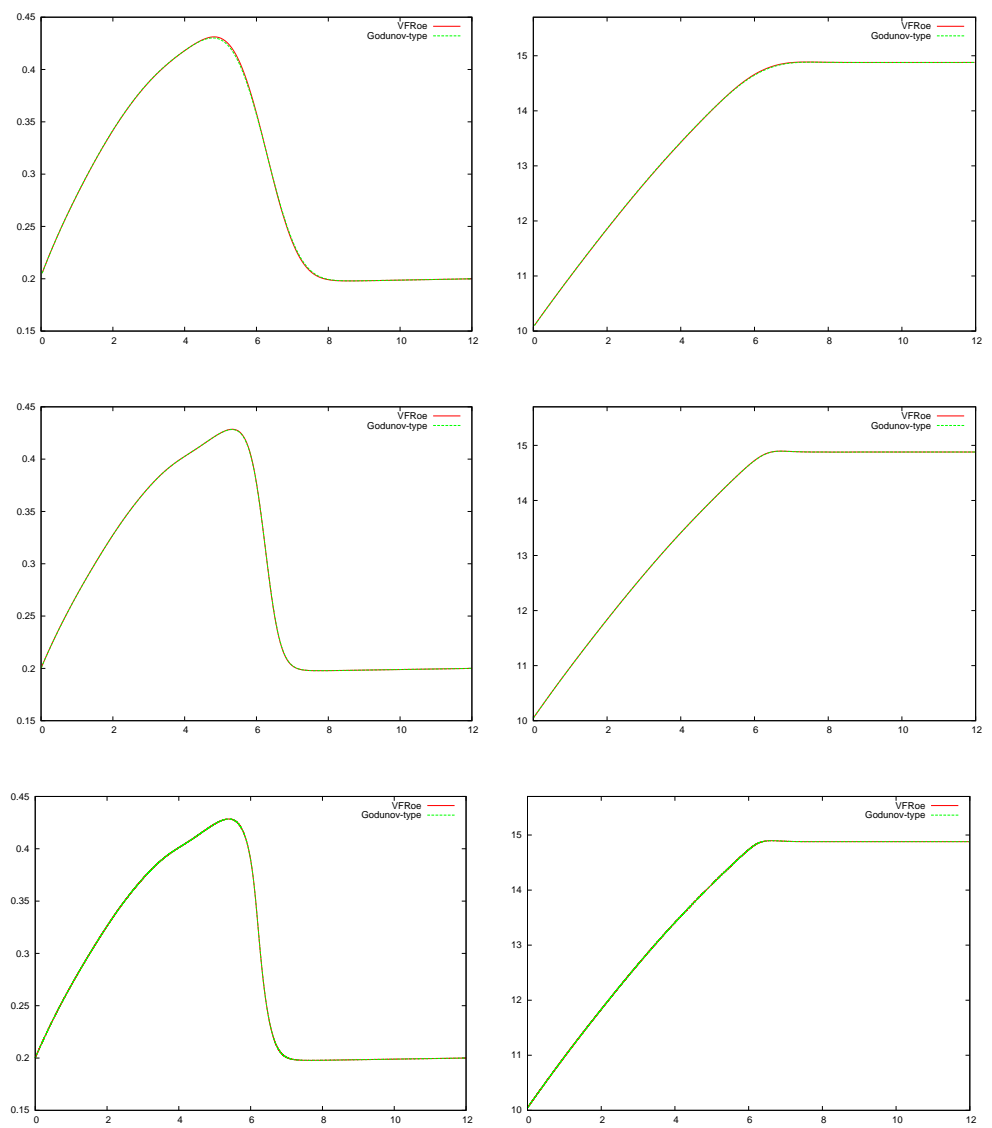

Figure 10: Comparison between numerical solutions of Test 4 at time $t=0.5$ given by the VFRoe scheme and the Godunov-type scheme and for several mesh sizes. On the left : $\alpha_{2}$, on the right : $u_{1}$. From the top to the bottom : 200-point mesh, 2000-point mesh and 20000-point mesh.
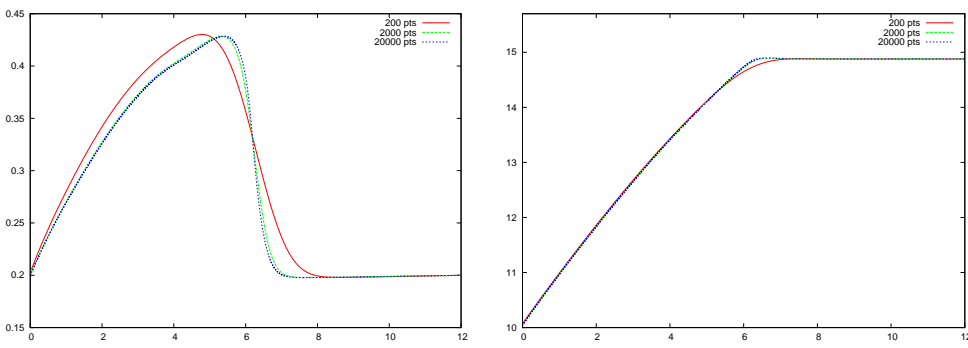

Figure 11: Comparison between numerical solutions of Test 4 at time $t=0.5$ given by the Godunov-type scheme and for several mesh sizes. On the left : $\alpha_{2}$, on the right : $u_{1}$. 
to phase vanishing, we have modified in our code, at each time iteration, the updated values of both velocities $u_{1}$ and $u_{2}$ and set them to $u_{1}=u_{2}=0$ as soon as either $\alpha_{1}$ or $\alpha_{2}$ is less than a threshold $\eta$. Hereafter, $\eta=10^{-5}$.

On Fig. 12, we give the numerical profile of the void fraction $\alpha_{1}$ at different intermediate times and for a mesh made of 400 cells. The time scale is taken to be $\tau_{p}=510^{-3} s$ in the pressure relaxation term. We observe that the solutions at times $t=1.2$ and $t=1.4$ are identical so that the stationary profile of $\alpha_{1}$ seems to be reached. We also note that the final void fraction discontinuity is not located at the middle point $x=3.75$ of the domain due to the compressibility effects.

On Fig. 13, we plot the solution obtained with $\tau_{p}=510^{-4} s$ and a 4000-cell mesh. The pressure relaxation time is thus faster and the mesh finer (the latter turned out to be necessary to get the numerical stability of the algorithm). We observe that the stationary profile appears to be attained more quickly.

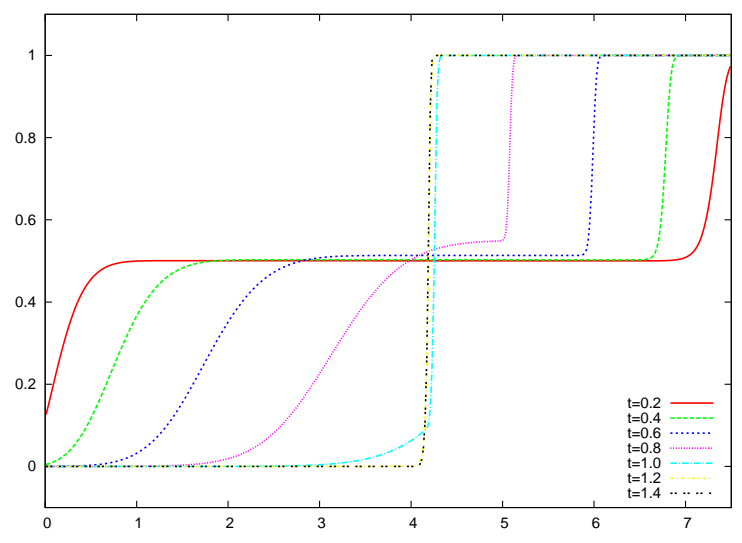

Figure 12: Numerical profile of the void fraction $\alpha_{1}: \tau_{p}=510^{-3} s$ and 400 cells

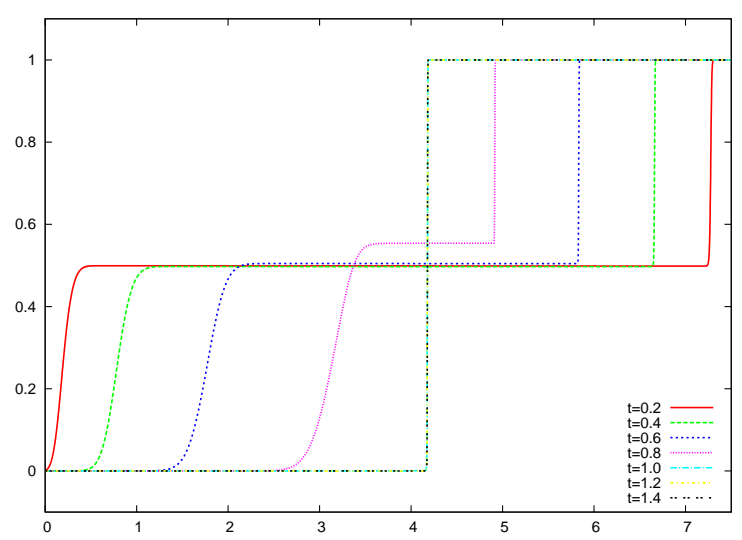

Figure 13: Numerical profile of the void fraction $\alpha_{1}: \tau_{p}=510^{-4} s$ and 4000 cells 


\subsubsection{Test 6 : velocities desequilibrium}

This test case corresponds to the evolution of a two-phse flow in a duct of uniform section and $1 \mathrm{~m}$ length. In this case, the external forces are assumed to be negligeable but we consider now a non zero interfacial drag force given by

$$
K(\mathbf{U})=\frac{1}{8} C_{D} a^{i n t} \rho_{1}\left|u_{1}-u_{2}\right| .
$$

In this formula, the coefficients

$$
C_{D}=0.5, \quad a^{i n t}=\frac{3\left(1-\alpha_{1}\right)}{r_{b}}, \quad r_{b}=2.510^{-4}
$$

represent a drag coefficient, an interfacial area and a mean bubbles radius respectively. Then, index 1 (resp. 2) is sort of a liquid (resp. vapor) phase.

The pressure relaxation time is taken to be $\tau_{p}=0.510^{-5}$.

Theoretical boundary conditions are such that at the inlet $x_{\min }=0$ we impose a non zero relative velocity such that $u_{1}=1 \mathrm{~m} / \mathrm{s}$ and $u_{2}=1.5 \mathrm{~m} / \mathrm{s}$, the void fraction $\alpha_{1}=0.8$ and the density values $\rho_{1}=10^{3} \mathrm{~kg} / \mathrm{m}^{3}$ and $\rho_{2}=1 \mathrm{~kg} / \mathrm{m}^{3}$. At the outlet $x_{\max }=1$, we only impose the pressures $p_{1}=p_{2}=101300 \mathrm{~Pa}$. From a numerical point of view, the fictitious states are such that

$$
\alpha_{1, \min }=0.8, \quad\left(\rho_{1}, u_{1}\right)_{\min }=\left(10^{3}, 1\right), \quad\left(\rho_{2}, u_{2}\right)_{\min }=(1,1.5)
$$

and

$$
p_{1, \max }=p_{2, \max }=101300 .
$$

Classical Neumann numerical boundary conditions are applied to define the others quantities.

Initial conditions are taken to be uniform in the computational domain and such that $u_{1}=u_{2}=0$, the others variable been chosen in agreement with the boundary conditions.

Our objective here is to test the ability of the method to compute the stationary solution associated with such a stiff source terms problem. First, due to the interfacial drag force, the relative velocity $u_{r}=u_{2}-u_{1}$ is expected to tend to zero very fast along the pipe (see [31]). Then, due to the relaxation pressure term, the relative pressure $p_{r}=p_{2}-p_{1}$ is expected to tend to zero as well. From the void fraction evolution equation, $\alpha_{1}$ is thus expected to become constant very fast along the pipe, as well as the pressures $p_{1}$ and $p_{2}$ (recall that the gravity field is not present here). From the mass and momentum equations, we easily see that the same happens for the densities and the partial velocities. We plot on Fig. 14 and 15 the stationary profiles given by a 2000-point mesh. We can observe that they are in good agreement with the expected behavior.

\subsubsection{Test 7 : vertical bubbly column}

This test case corresponds to the evolution of a two-phase flow in a vertical column of $1 m$ length. In this case, both the gravity $g=-9.81 \mathrm{~m} / \mathrm{s}^{2}$ and a non zero interfacial drag force are considered. The latter is given by the same formula as in the previous test case. In the pressure relaxation source term, the 

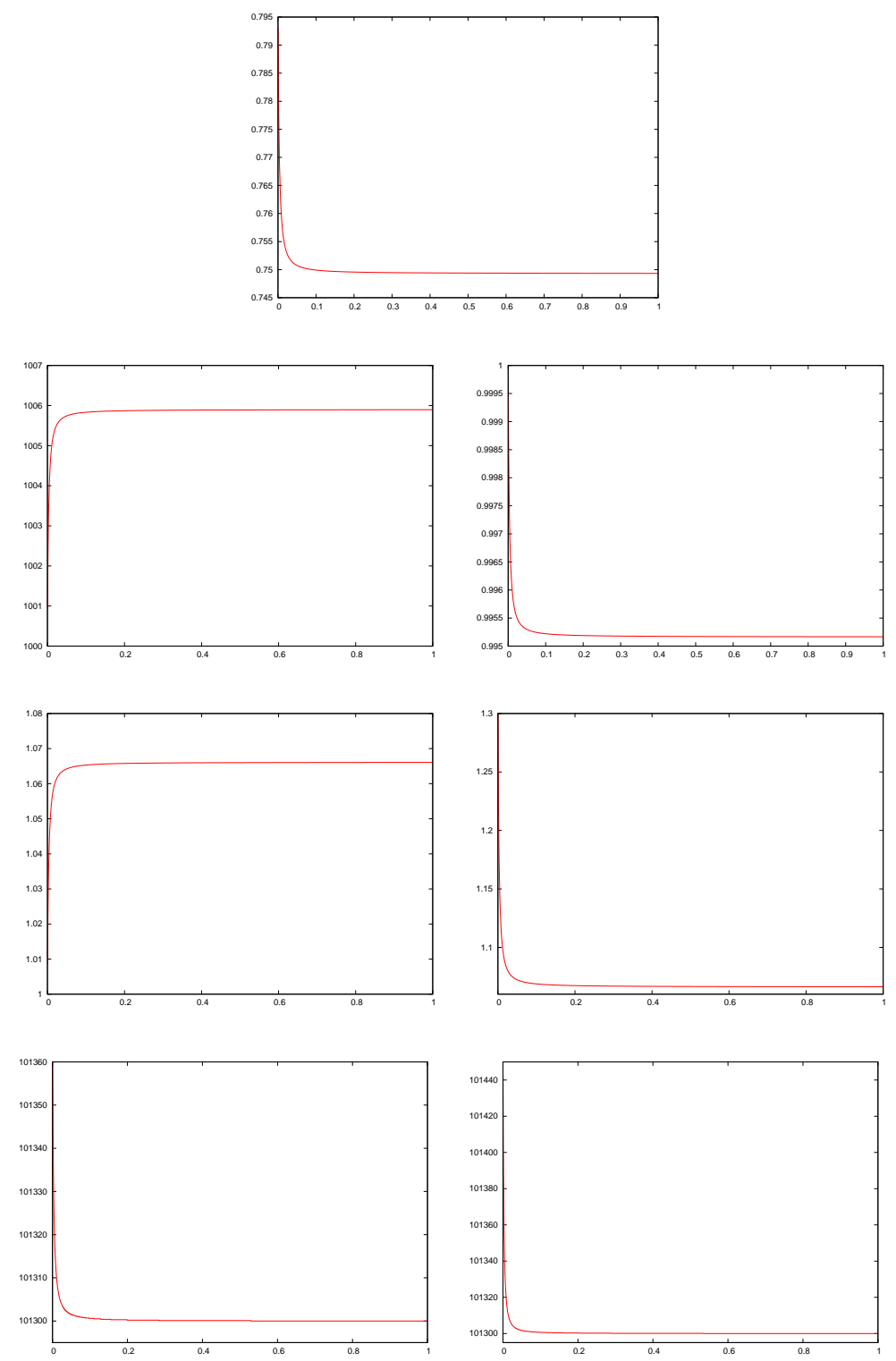

Figure 14: Stationary solution of Test 6 . From the top to the bottom right : $\mathrm{x}$ versus $\alpha_{1}, \rho_{1}, \rho_{2}, u_{1}, u_{2}, p_{1}, p_{2}$ 

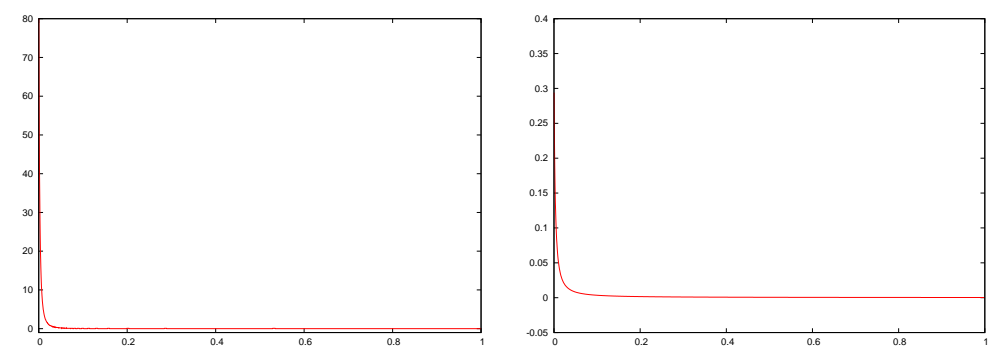

Figure 15: Stationary solution of Test 6. Close-up. From the left to the right : $\mathrm{x}$ versus $p_{r}=p_{2}-p_{1}, u_{r}=u_{2}-u_{1}$

relaxation time is taken to be $\tau_{p}=1.10^{-4}$.

Theoretical boundary conditions are such that at the inlet $x_{\min }=0$, we impose a non zero relative velocity such that $u_{1}=5 \mathrm{~m} / \mathrm{s}$ and $u_{2}=15 \mathrm{~m} / \mathrm{s}$, the void fraction $\alpha_{1}=0.97$, the density values $\rho_{1}=10^{3} \mathrm{~kg} / \mathrm{m}^{3}$ and $\rho_{2}=1 \mathrm{~kg} / \mathrm{m}^{3}$, and the mixture pressure $p=15510^{5} \mathrm{~Pa}$. At the outlet $x_{\max }=1$, we only impose the mixture pressure $p=15010^{5} \mathrm{~Pa}$.

From a numerical point of view, the fictitious states are such that

$\alpha_{1, \min }=0.97, \quad\left(\rho_{1}, u_{1}, p_{1}\right)_{\min }=\left(10^{3}, 5,15510^{5}\right), \quad\left(\rho_{2}, u_{2}, p_{2}\right)_{\min }=\left(1,15,15010^{5}\right)$

and

$$
p_{1, \max }=p_{2, \max }=15010^{5} .
$$

Classical Neumann numerical boundary conditions are applied to define the others quantities.

Initial conditions are taken to be uniform in the computational domain and in agreement with the inlet boundary conditions for all the variables except the pressures that are defined from the oulet boundary values.

We plot on Fig. 16 the stationary profiles given by a 400-point mesh. Our objective here is to assess the capability of our algorithm to restore at the numerical level a specific asymptotic behavior of the solutions of the two-pressure diphasic model under consideration in this paper. More precisely, it is recalled in section 2.3 that if the time scales of the pressure relaxation and drag force source terms involved in the model are much smaller than the other characteristic time scales of the system, and provided that we have a balance of the gradient of pressure with the external forces, which writes here

$$
\partial_{x} p=-\rho g, \quad p=\alpha_{1} p_{1}+\alpha_{2} p_{2}, \quad \rho=\alpha_{1} \rho_{1}+\alpha_{2} \rho_{2},
$$

then the solutions behave like the solutions of a drift-flux model. Such a model is governed by conservation laws on mixture density, mixture momentum, mixture total energy and mass fraction of one of the two phases, and involves a closure law on the relative velocity $u_{r}=u_{2}-u_{1}$. From (24), (94) and (95) the corresponding drift law is

$$
\frac{1}{8} C_{D} a^{i n t} \rho_{1}\left|u_{r}\right| u_{r}=\alpha_{1} \alpha_{2}\left(\rho_{2}-\rho_{1}\right) g .
$$


Note that in the present test case, we have $u_{1}<u_{2}$ and $\rho_{2}<<\rho_{1}$. We then easily get $u_{r}>0$, so that taking into account the values of the different coefficients,

$$
u_{r}^{2}=-\frac{1}{750} \alpha_{1}\left(1-\frac{\rho_{2}}{\rho_{1}}\right) g
$$

(recall that $g<0$ )

We can observe on Fig. 17 that this asymptotic drift law is numerically satisfied, as well as the expected balance between the gradient of pressure and the gravity field.

\section{Acknowledgments}

The authors are very grateful to F. Coquel and N. Seguin for the very beneficial discussions we had on this work.

\section{References}

[1] A. Ambroso, C. Chalons, F. Coquel, T. Galié. Relaxation and numerical approximation of a two fluid two pressure diphasic model. Mathematical Modelling and Numerical Analysis (M2AN), vol 43, pp 1063-1097, (2009).

[2] A. Ambroso, C. Chalons, F. Coquel, T. Galié, E. Godlewski, P.-A. Raviart and N. Seguin. The drift-flux asymptotic limit of barotropic two-phase twopressure models. Comm. Math. Sci., vol 6, pp 521-529, (2008).

[3] N. Andrianov, Analytical and numerical investigation of two-phase flows, PhD thesis, Univ. Magdeburg (2003).

[4] N. Andrianov, R. Saurel and G. Warnecke. A simple method for compressible multiphase mixtures and interfaces. Technical Report 4247, INRIA, (2001).

[5] N. Andrianov and G. Warnecke. The Riemann problem for the BaerNunziato two-phase flow model. Journal of Computational Physics, vol 195, pp 434-464 (2004).

[6] M.R. Baer and J.W. Nunziato, A two phase mixture theory for the deflagration to detonation (DDT) transition in reactive granular materials. Int. J. Multiphase Flows, Vol. 12(6), (1986), pp. 861-889.

[7] F. Bouchut, Nonlinear stability of finite volume methods for hyperbolic conservation laws, and well-balanced schemes for source. Frontiers in Mathematics Series, Birkhauser, 2004.

[8] T. Buffard, T. Gallouët and J.M. Hérard, A sequel to a rough Godunov scheme. Application to real gas flows, Computers and Fluids, Vol. 29(7), (2000), pp. 813-847.

[9] C.E. Castro and E.F. Toro, A Riemann solver and upwind methods for a two-phase flow model in nonconservative form, International Journal of Numerical Methods in Fluids, Vol. 50, (2006), pp. 275-307. 

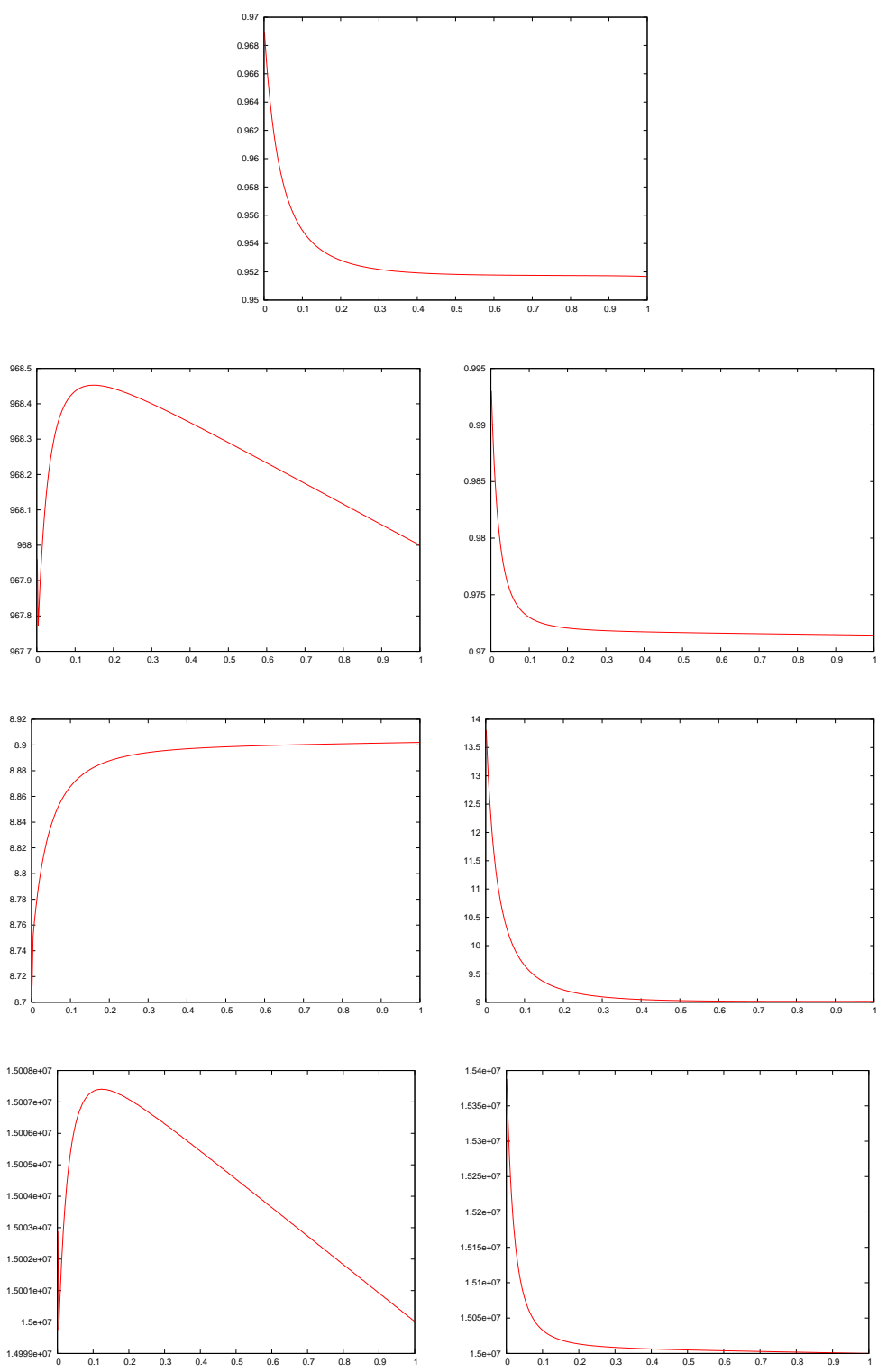

Figure 16: Stationary solution of Test 7 . From the top left to the bottom right : x versus $\alpha_{1}, \rho_{1}, \rho_{2}, u_{1}, u_{2}, p_{1}, p_{2}$ 

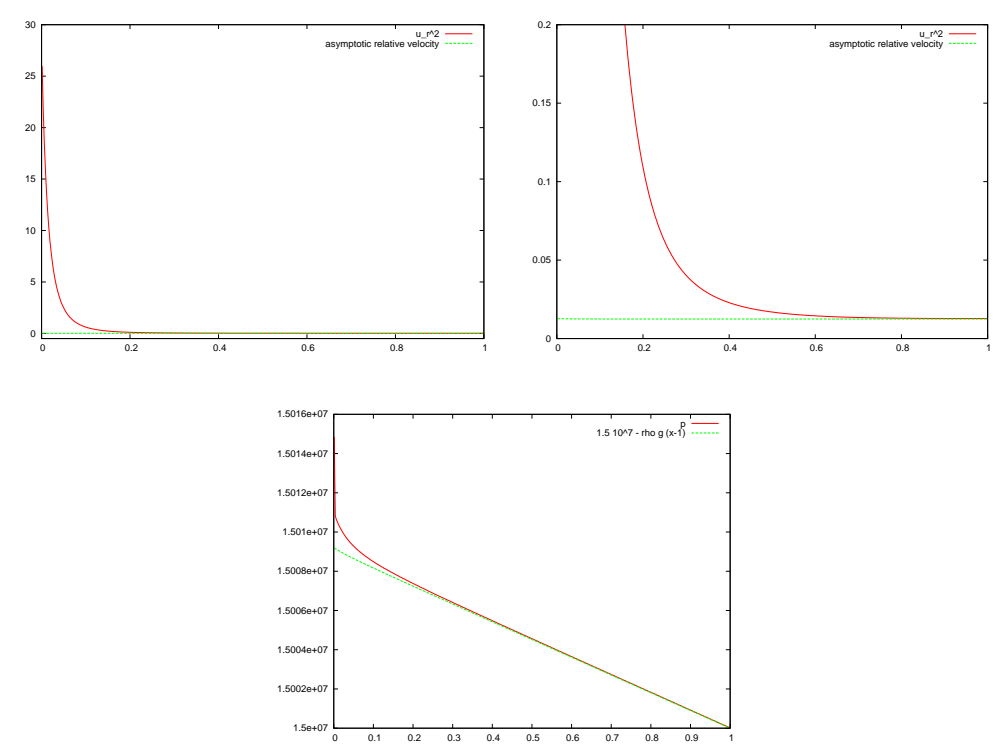

Figure 17: Stationary solution of Test 7 : relative velocity $u_{r}^{2}$ and mixture pressure $p$. From the top left to the bottom : $\mathrm{x}$ versus $u_{r}^{2}, u_{r}^{2}$ (close-up), $p$

[10] C. Chalons, F. Coquel, E. Godlewski, P.-A. Raviart and N. Seguin, Godunov-type schemes for hyperbolic systems with parameter dependent source. The case of Euler system with friction, to appear in M3AS, Mathematical Models and Methods in Applied Sciences, 2010.

[11] C. Chalons and J.-F. Coulombel, Relaxation approximation of the Euler equations. J. Math. Anal. Appl., vol 348, no. 2, pp 872-893 (2008)

[12] F. Coquel, K. El Amine, E. Godlewksi, B. Perthame and P. Rascle. A numerical method using upwind schemes for the resolution of two-phase flows. Journal of Computational Physics, vol 136, pp 272-288 (1997).

[13] F. Coquel, T. Gallouët, J.M. Hérard and N. Seguin, Closure laws for a twophase two-pressure model, Comptes Rendus Mathématique, Vol. 334, (2002), pp. 927-932.

[14] S. Dellacherie. Relaxation schemes for the multicomponent Euler system. Math. Model. and Num. Anal., vol 37(6), pp 909-936 (2003).

[15] V. Deledicque and M.-V. Papalexandris. An exact Riemann solver for compressible two-phase flow models containing non-conservative products. Journal of Computational Physics, vol 222, pp 217-245 (2007).

[16] P. Embid and M. Baer, Mathematical analysis of a two-phase continuum mixture theory, Contin. Mech. Thermodyn. Vol. 4, no. 4, (1992), pp. 279312 .

[17] T. Galié. Couplage interfacial de modèles en dynamique des fluides. Application aux écoulements diphasiques. $\mathrm{PhD}$ thesis, Université Pierre et Marie Curie - Paris 6, France, (2009). 
[18] G. Gallice. Solveurs simples positifs et entropiques pour les systèmes hyperboliques avec terme source. (French) [Entropic Godunov-type schemes for hyperbolic systems with source term.] C. R. Math. Acad. Sci. Paris, 334 (8), pp 713-716 (2002).

[19] G. Gallice. Schémas positifs et solveurs de Riemann simples entropiques pour des systèmes hyperboliques avec terme source : application à la dynamique des gaz avec gravité. Preprint MAB-LRC 02.05, Université Bordeaux 1, France.

[20] G. Gallice. Positive and entropy stable Godunov-type schemes for gas dynamics and MHD equations in Lagrangian or Eulerian coordinates. Numer. Math., 94(4), pp 673-713 (2003).

[21] T. Gallouët, J.-M. Hérard and N. Seguin. Numerical modeling of two-phase flows using the two-fluid two-pressure approach. Mathematical Models and Methods in Applied Sciences (M3AS), vol 14(5), pp 663-700 (2004).

[22] S. Gavrilyuk and R. Saurel, Mathematical and numerical modeling of twophase compressible flows with micro-inertia., J. Comput. Phys., Vol. 175, (2002), pp. 326-360.

[23] J. Glimm, D. Saltz and D.H. Sharp, Two phase flow modelling of a fluid mixing layer. J. Fluid Mech., Vol. 378, (1999), pp. 119-143.

[24] H. Guillard and A. Murrone, A five equation reduced model for compressible two phase flow problems, INRIA report, 4778, ISSN 0249-6399 (2003).

[25] V. Guillemaud, Modélisation et simulation numérique des écoulements diphasiques par une approche bifluide à deux pressions, Thèse, Université de Provence, Aix-Marseille 1, (2007).

[26] A. Harten, P.-D. Lax and B. Van Leer, On upstream differencing and Godunov-type schemes for hyperbolic conservation laws, SIAM Rev., vol 25, pp 35-61 (1983).

[27] T. Hibiki and M. Ishii, One-dimensional drift-flux model and constitutive equations for relative motion between phases in various two-phase flows regime, Int. J. of Heat and Mass Transfer, Vol 46, 2003, pp. 4935-4948.

[28] M. Ishii and N. Zuber, Drag coefficient and relative velocity in bubbly, droplet or particulate flows, AIChE J., Vol. 25, No. 5, 1979, pp. 843-855.

[29] A.K. Kapila, S.F. Son, J.B. Bdzil, R. Menikoff and D.S. Stewart, Two phase modeling of DDT: structure of the velocity-relaxation zone. Phys. Fluids, Vol. 9(12), (1997), pp. 3885-3897.

[30] S. Karni, E. Kirr, A. Kurganov and G. Petrova, Compressible two-phase flows by central and upwind schemes, ESAIM: Mathematical Modelling and Numerical Analysis (M2AN), Vol. 38(3), (2004), pp. 477-493.

[31] A. Kumbaro and A. Chanoine. Numerical benchmarks computed with the OVAP code. Technical Report DM2S/SFME/LETR/RT/05-049/A, CEASaclay, (2005). 
[32] P.G. LeFloch Entropy weak solutions to nonlinear hyperbolic systems in nonconservative form, Comm. Part. Diff. Equa. Vol. 13, pp. 669-727 (1988).

[33] P.G. LeFloch Shock waves for nonlinear hyperbolic systems in nonconservative form, Institute for Math. and its Appl., Minneapolis Preprint \# 593, (1989).

[34] S.T. Munkejord, Comparison of Roe-type methods for solving the two-fluid model with and without pressure relaxation, Computers and Fluids, Vol. 36, (2007), pp. 1061-1080.

[35] V.-H. Ransom. Numerical benchmark tests. Multiphase Science and Technology, Hemisphere publishing corporation, G.F. Hewitt, J.M. Delhaye \& N. Zuber edition, (1987).

[36] V.V Rusanov, Calculation of interaction of non-steady shock waves with obstacles, J. Comp. Math. Phys., USSR, Vol. 1, (1961), pp. 267-279.

[37] R. Saurel and R. Abgrall, A multiphase Godunov method for compressible multifluid and multiphase flows, J. Comput. Phys., Vol. 150, (1999), pp. $425-467$.

[38] R. Saurel and R. Abgrall, Discrete equations for physical and numerical compressible multiphase mixtures, J. Comput. Phys., Vol. 186, (2003), pp. 361-396.

[39] R. Saurel and O. Lemetayer, A multiphase model for compressible flows with interfaces, shocks, detonation waves and cavitation, J. Fluid Mech., Vol. 431, (2001), pp. 239-271.

[40] D.-W. Schwendeman, C.-W. Wahle and A.-K. Kapila. The Riemann problem and a high-resolution Godunov method for a model of compressible twophase flow. Journal of Computational Physics, vol 212, pp 490-526 (2006).

[41] B. Stewart and B. Wendroff, Two-phase flow : models and methods, $J$. Comput. Phys., Vol. 56, (1984), pp. 363-409.

[42] S.-A. Tokareva and E.-F. Toro, HLLC-type Riemann solver for the BaerNunziato equations of compressible two-phase flow, J. Comput. Phys., to appear, (2010) 